University of New Mexico

UNM Digital Repository

Mechanical Engineering ETDs

Engineering ETDs

7-11-2013

\title{
Deformation Localization in Constrained Layers of Metallic Glasses: A Parametric Modeling Analysis
}

Daneil Rogers

Follow this and additional works at: https://digitalrepository.unm.edu/me_etds

\section{Recommended Citation}

Rogers, Daneil. "Deformation Localization in Constrained Layers of Metallic Glasses: A Parametric Modeling Analysis." (2013). https://digitalrepository.unm.edu/me_etds/69

This Thesis is brought to you for free and open access by the Engineering ETDs at UNM Digital Repository. It has been accepted for inclusion in Mechanical Engineering ETDs by an authorized administrator of UNM Digital Repository. For more information, please contact disc@unm.edu. 
Daniel Rogers

Candidate

Mechanical Engineering

Department

This thesis is approved, and it is acceptable in quality and form for publication:

Approved by the Thesis Committee:

Dr. Yu-Lin Shen , Chairperson

Dr. Rafiqul A. Tarefder

Dr. Jesse Thomas 


\title{
Deformation Localization in Constrained Layers of Metallic Glasses: A Parametric Modeling Analysis
}

by

\author{
Daniel N. Rogers
}

B.S., Mechanical Engineering, University of New Mexico, 2011

\section{THESIS}

Submitted in Partial Fulfillment of the

Requirements for the Degree of

\author{
Master of Science \\ Mechancial Engineering
}

The University of New Mexico

Albuquerque, New Mexico

May, 2013 
(C)2013, Daniel N. Rogers 


\section{Dedication}

To my parents, David and Darlene, for their support and encouragement throughout my college career. 


\section{Acknowledgments}

I would like to thank my advisor, Professor Yu-Lin Shen, for his support and guidance through out my graduate research. His expertise was inavluable at not only getting our research published but also further developing my undertanding of engineering.

I am paticularly grateful for the opportunity to use Sandia National Laboratories FEA code Sierra/Solidmechaics provided to me in part by Dr. Joe Jung. Without him taking me on as an intern in his department at Sandia Labs I would have not have had the skills/tools necessary to complete this thesis.

I would like to thank my mentor and colleague Dr. Jesse Thomas for not only serving on my thesis committee but also his help and advice in helping me better understand computaional solid mechanics. Finally, I want to thank Dr. Rafiqul A. Tarefder for also serving on my thesis committee. 


\title{
Deformation Localization in Constrained Layers of Metallic Glasses: A Parametric Modeling Analysis
}

by

\author{
Daniel N. Rogers
}

B.S., Mechanical Engineering, University of New Mexico, 2011

M.S., Mechanical Engineering, University of New Mexico, 2013

\begin{abstract}
Localized plastic deformation known as shear banding is a prominent feature in metallic glasses. In this study we perform parametric three-dimensional finite element analyses, using primarily a thin layer of metallic glass on top of a cylindrical base, to study how physical constraint can affect this localized form of deformation and the corresponding macroscopic stress-strain response. Random perturbation points are added to the metallic glass model to facilitate the formation of shear bands. The modeling result suggests that the mechanical behavior of metallic glasses can be significantly influenced by the geometrical confinement. Under nominally uniaxial compressive loading, a lower thickness-to-diameter ratio results in higher plastic flow stresses. Shear bands tend to concentrate in regions away from the interface with the base material. The findings provide a mechanistic rationale for experimental observations based on the micropillar compression test. The deformation pattern in a multilayered metallic glass structure as well as the deformation pattern in a metallic glas beam subjected to four point bending are also examined.
\end{abstract}




\section{Contents}

List of Figures $\quad$ ix

List of Tables $\quad$ xiii

1 Introduction 1

2 Numerical Model $\quad 6$

2.1 Micro Pillar Models . . . . . . . . . . . . . . . . . . . . . 6

2.2 Beam Model . . . . . . . . . . . . . . . . . . . 10

3 Mesh Convergence Study 11

3.1 Background ........................... 11

3.2 Numerical Model . . . . . . . . . . . . . . . . . . . . . 12

3.3 Results ......................... 14

3.4 Conclusion . . . . . . . . . . . . . . . . . . 17

4 Results and Discussion $\quad 18$ 
Contents

4.1 Stand-alone Metallic Glass Cylinder . . . . . . . . . . . . . . . . . . . 18

4.2 Cylinders Constrained by Base Material . . . . . . . . . . . . . . . . 20

4.2.1 Comparison with Experimentation . . . . . . . . . . 25

4.3 Multilayered Structure . . . . . . . . . . . . . . . . . 27

4.3.1 Comparison with Experimentation . . . . . . . . . . . 31

4.4 Effect of Taper . . . . . . . . . . . . . . . . . . . . . . . 33

4.5 Four Point Bending . . . . . . . . . . . . . . . . . . . 34

4.5.1 Comparison with Experimentation . . . . . . . . . . . 35

5 Conclusions and Suggested Future Work 36

5.1 Implications . . . . . . . . . . . . . . . . . . . . . . . . 38

$\begin{array}{ll}\text { References } & 39\end{array}$ 


\section{List of Figures}

1.1 Experimental Images of shear band formation (a) Shear bands for less ductile $Z r_{65} C u_{15} N i_{10} A l_{10}$ BMG (b) Shear bands for more ductile $C u_{47} Z r_{47} A l_{5}$ BMG (c) Mangnified view of more ductile metallic glass where intersection, arrests and branching of the shear bands are shown $[12] \ldots \ldots \ldots \ldots \ldots \ldots$

2.1 Schematics of the numerical models subjected to compressive loading: (a) a stand-alone metallic glass cyliner, (b) a layer of metallic glass above a supporting base, and (c) multilayered metallic glass/reference metal structure . . . . . . . . . . . . . . . . 7

2.2 Schematic of the distribution of perturbation points in the standalone metallic glass model . . . . . . . . . . . . . . . . . . .

2.3 Schematic of the numerical beam model subjected to four point bending. . . . . . . . . . . . . . . . . . . . 10

3.1 Uniform mesh refinement of single hex element . . . . . . . . . . . . 13

3.2 Stand-alone metallic glass mesh refinements: (a) mesh 1, (b) mesh 2, (c) mesh 3, (d) mesh 4, (e) mesh 5 . . . . . . . . . . . . . . . 14 


\section{List of Figures}

3.3 Stand-alone metallic glass mesh refinement - Stress vs. Strain . . . . 15

3.4 Yield stress for various levels of mesh refinement . . . . . . . . . . . 16

4.1 Simulated stress-strain curves for the stand-alone metallic glass cyliners with aspect ratios $0.2,0.4,0.6,0.8$ and $1.0 \ldots \ldots$

4.2 Contours of equivalent plastic strain in the stand-alone metallic glass cylinders with aspect ratio (a) 1.0 and (b) 0.6, at the same macro-

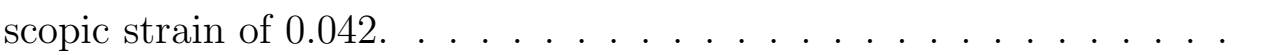

4.3 Simulated stress-strain curves for the metallic glass cyliners, with aspect ratios $0.2,0.4,0.6,0.8$ and 1.0 , when they are attached to a Si base......................... 20

4.4 Simulated $1 \%$ offset yield stress as a function of the aspect ratio of the Si-attached metallic glass. . . . . . . . . . . . . . . . 21

4.5 Contours of equivalent plastic strain in the Si base-attached metallic glass cylinders with aspect ratio (a) 1.0 and (b) 0.6, at the same macroscopic strain of $0.042 \ldots \ldots \ldots \ldots$

4.6 Simulated $1 \%$ offset yield stress as a function of the aspect ratio of the Si-attached metallic glass. In addition to the standard model of $1 \%$ perturbation points, two additional cases, with 0 and $10 \%$ perturbation points, are included. . . . . . . . . . . . 23

4.7 Contours of equivalent plastic strain in the Si base-attached metallic glass free of any perturbation points, with aspect ratios (a) 1.0 and (b) 0.6 at the same macroscopic strain of $0.042 \ldots \ldots 24$ 


\section{List of Figures}

4.8 Contours of equivalent plastic strain in the "hard metal" base-attached metallic glass with aspect ratios (a) 1.0 and (b) 0.6 at the same macroscopic strain of $0.042 \ldots \ldots \ldots . \ldots . \ldots 25$

4.9 (a)-(e) SEM micrographs showing the TFMG/Si composite micropillars with different sizes and shapes. Note that a sketched profile of the micropillar is superimposed on the corresponding SEM micrograph for clarity. $[2] \ldots \ldots \ldots 26$

4.10 The variation of the yield strengths of the TFMGs obtained from microcompression with their $t_{f} / D$ ratios. $[2,17] \ldots$. . . . . . . . 27

4.11 Simulated stress-strain curve of the multilayer model. Also included is the stand-alone metallic glass model of the same overall dimension (the case of $\mathrm{t} / \mathrm{D}=1$ in Fig. 2). . . . . . . . . . . . . . 28

4.12 Contour plot of equivalent plastic strain in the multilayer model when the macroscopic strain is at 0.042 . . . . . . . . . . . . . 29

4.13 Multilayer metallic glass model with built in hardening response: (a) $10 \%$ Strain Hardening, (b) 25\% Strain Hardening . . . . . . . . . . . 29

4.14 Multilayer metallic glass models with built in hardening response. The EQPS scale has been adjusted for each model to better show the development of shear bands. (a) 10\% Strain Hardening, (b) 25\% Strain Hardening, (c)75\% Strain Hardeding and (d) 100\% Strain Hardening ....................... . . . . . . . . .

4.15 Contour plot of equivalent plastic strain in the multilayer model when the macroscopic strain is at 0.042

4.16 SEM micrograph showing $\mathrm{ZrCu} / \mathrm{Cu}$ five-layer pillar compressed to a predetermined displacment of 300nm [37] . . . . . . . . . . . . . 32 


\section{List of Figures}

4.17 Contour plot of equivalent strain in the Si-base attached metallic glass model, with a $3^{\circ}$ taper, at the macroscopic compressive strain of $0.042 \ldots \ldots \ldots \ldots \ldots$

4.18 Contour plots of equivalent plastic strain in metallic glass due to bending deformtation: (a) Front view (b) Top view (c) Bottom view. 34

4.19 SEM micrographs of MG/Ti bilayer-coated BMG surface after bending to failure (a surface strain of 13.7\%): (a) $50^{\circ}$ tilted and (b) side view. $[32] \ldots \ldots \ldots \ldots \ldots$ 


\section{List of Tables}

3.1 Stand-alone metallic glass parameters for mesh convergence study. . 16 


\section{Chapter 1}

\section{Introduction}

Finite element analysis (FEA) has become a common technique used to analyze and examine the reaction of solid materials to internal and external forces. In recent years the development of nano- and micro- scale devices has led to an expanded interest in the deformation of materials at small scales [1]. Due to the cost and time necessary to develop these small scales devices it becomes increasingy difficult to perform systematic experimental research on material properties at such small scales. Through the use of computational experimentation the mechanical properties of common and emerging materials can be investigated. Amorphous metals known as metallic glasses have recently come under investigation for their potential use as a structural material in future engineering applications [2].

Metallic glasses (MGs), or amorphous metallic alloys, are non- crystalline metals which lack long-range atomic periodicity because they are generally formed with fast quench rates for the retention of the glassy state from the melt [3]. MGs are considered to be non-equilibrium materials. Due to the absence of grain boundaires and a distorted atomic structure, MG's have been shown to have exceptional material properties. MG's usually display an exceptionally higher yield strength than 


\section{Chapter 1. Introduction}

most crystalline metals. Other superior characterstics of MG's include the materials harndess, elastic limit ( $2 \%$ ) and good wear resistance.

The unique mechanical and functional properties displayed by bulk metallic glasses have sparked widespread interests in the materials research community [4-10]. These amorphous alloys generally exhibit elastic stiffness comparable to those of conventional engineering alloys, but their strengths at ambient temperature may be significantly higher. The absence of crystallinty in the microstructure, however, results in limited deformability. Dislocation slip, mechanical twinning and other deformation mechanisms associated with crystalline structures are no longer applicable. At below the glass transition temperature, plastic deformation occurs in a highly inhomogeneous manner via the formation and propagation of shear bands.

A shear band is typically a 10-100 nm-thick zone embedded within the relatively un-deformed matrix. Once shear starts locally, it tends to concentrate there and extends along a geometrically favorable path. In other words, the shear band becomes a weaker region than its surrounding. This is a manifestation of the work softening phenomenon inside a shear band. The accumulation of shear strain in an individual shear band may be as high as 10, although the overall ductility of the metallic glass specimen is still relatively small [11]. Figure 1.1 shows some SEM micrographs of shear bands that have formed in a bulk metallic glass specimen that has been loaded to failure.

Bulk metallic glasses (BMGs) have been a focus of many studies over the years because of the amorphous alloys unique properties. The first studies performed on BMGs were done in the 1960's and 1970's (Klement et al. [13], Chen and Turnbell [14] and Chen [15]). The first creation of metallic glasses was reported by Klement et al. [13] at Caltech, USA in 1960. They discovered that if a molten metal alloy made up of $A u_{75} S i_{25}$ is undercooled uniformly and rapdily $\left(10^{5}-10^{6} \mathrm{Ks}^{-1}\right)$ the heterogeneous atoms do not have enough time or energy to rearrange and establish 
Chapter 1. Introduction

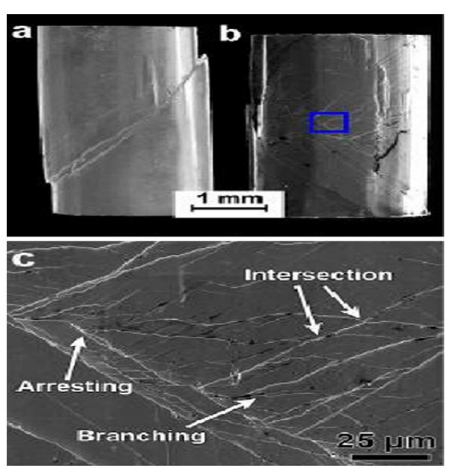

Figure 1.1: Experimental Images of shear band formation (a) Shear bands for less ductile $Z r_{65} C u_{15} N i_{10} A l_{10}$ BMG (b) Shear bands for more ductile $C u_{47} Z r_{47} A l_{5}$ BMG (c) Mangnified view of more ductile metallic glass where intersection, arrests and branching of the shear bands are shown [12]

crystal nucleation. The initial work doned showed that the process of nucleation and crystalline growth phase could be kinetically bypassed in some alloy melts producing a frozen liquid known as metallic glass. The initail metallic glasses that were formed were created using rapid solidification methods limiting the metallic glass form to thin strips or splats, severley limiting the uses they could be applied to. The need to produce metallic glass in bulk form led researchers Chen and Turnbell [14] to understand the governing principles needed to form metallic glasses.

Chen and Turnbell were able to show similarities between metallic glasses and other non-metallic glasses such as silicates, ceramic glasses, and polymers. They showed that, a glass transition in conventional glass-forming melts could also be observed in rapidly quenched metallic glasses. From thermodynamic and kinetic considerations of crystallisation involving nucleation growth, they predicted that a ratio, referred to as the reduced glass transition temperature $T_{r g}=T_{g} / T_{m}$ (of the glass transition temperature $T_{g}$ to the melting point $T_{m}$ of alloy) can be used as a criterion for determining the glass-forming ability (GFA) of an alloy. According to Turnbulls criterion, a metal liquid with $T_{g} / T_{m}=2 / 3$ starts to move very slow 


\section{Chapter 1. Introduction}

allowing the liquid metal to be easily under-cooled at a low cooling rate therefore creating the metallic glass state. From these governing principles the first Bulk Metallic Glass (BMG) capable of being cast into samples greater than $1 \mathrm{~mm}$ in size were produced. The composition of the metallic glass produced was based on the Pd$\mathrm{Cu}-\mathrm{Si}$ alloy system which had a very low cooling rate of $10 \mathrm{Ks}^{-1}$. Unfortunatly due to the high cost of the palladium in the alloy the pioneering works on metallic glass did not make a big impact in the materials science community. The breakthrough in BMG research came in the late 1980's with the discovery of multicomponet glass formers by Inoue at Tohoku University $[4,16]$.

The research team at Tohoku University was able to create new bulk glassy alloys that exhibit excellent glass forming ability and very low critical cooling rates for bulk glass formation without the use of exspenive noble metals like platinum and palladium. Inoue and his team showed that they were able to create metallic glasses with good GFA using Mg, Ln, Zr, Fe, Pd, Cu, Ti, Al and Ni based systems. Inoues efforts highlighted the fact that the correct choice of elemental constituents would lead to amorphous alloys even by using cooling rates employed in conventional industrial practices. By using slower cooling rates large peices of metallic glasses can be constructed making it possible to investigate the mechanical, physical and chemical properties of metallic glass. With increasing interest in developing and understanding these new types of amorphous metals researchers have begun making thin film metallic glass (TFMGs).

Metallic glasses in the form of deposited thin films have received great attention in recent years, due to their potential applications in emerging micro- and nano-scale devices $[3,17]$. As a result of their thin dimensions the flexibility can be improved, but deformation localization is still dominant once plastic yielding starts. In fact, the individual shear banding event may become more significant in affecting the mechanical property because of the small physical size. It has been reported that 


\section{Chapter 1. Introduction}

mechanical behavior of metallic glasses depends on their physical size [18-26]. While the atomic and structural origin of the intrinsic size effect has not been properly established, it is acknowledged that geometric confinement alone during mechanical testing of small-sized specimens can dictate the measured stress-strain response and deformation pattern in metallic glasses $[2,17,27]$.

For traditional crystalline materials capable of homogeneous plastic deformation, the geometric confinement effect is well recognized. For instance, plastic deformation disturbed by physical constraint in small metallic structures (such as films, lines and joints), bonded to substrates or other adjoining materials, has been widely examined $[1,28]$. The same effect on materials showing inherently localized deformation such as shear banding, on the other hand, is in need of further investigations. The present study is thus devoted to externally constrained plasticity in metallic glasses from a numerical modeling standpoint. Parametric finite element analyses are performed, utilizing a modeling scheme of a thin layer of metallic glass on an underlying cylindrical base (representative of the micropillar compression test [17]). The primary objectives of this thesis include:

- To provide mechanistic insight into interface-mediated localized deformation, through a simple modeling strategy applied to uniaxial compressive loading;

- To explore the apparent yield strength and shear band evolution as affected by the aspect ratio of the metallic-glass thin films;

- To compare the constrained deformation behavior in materials prone to shear banding and traditional homogeneous plasticity.

Where applicable, qualitative comparisons with reported experimental observations are made and the implications discussed. 


\section{Chapter 2}

\section{Numerical Model}

\subsection{Micro Pillar Models}

Three-dimensional finite element models were constructed. Figure 2.1(a) shows a schematic of the model consisting of only the cylindrical metallic glass specimen itself. The diameter $\mathrm{D}$ is fixed at $1 \mu \mathrm{m}$, and various thickness ( $\mathrm{t}$ ) values from 0.2 to $1 \mu \mathrm{m}$ are considered. Quasi-static compressive loading is applied through the prescribed displacement on the top face (initially at $\mathrm{z}=\mathrm{t}$ ). On the bottom face $(\mathrm{z}=0)$, movement along the $\mathrm{z}$-direction is prohibited but displacements in $\mathrm{x}$ and y are not constrained. This baseline case serves as a reference for contrasting with other models involving a support material (substrate) as shown in Fig. 2.1(b). Here a metallic glass layer is attached to a base, and the entire structure is subject to compressive deformation. Two base materials, silicon $(\mathrm{Si})$ and a hard metal (with hypothetical property described below), both with thickness (h) of $2 \mu \mathrm{m}$, are considered in this study. Figure 2.1(c) shows another model with an alternating metallic glass/reference metal structure. The thickness of each layer is kept at $0.2 \mu \mathrm{m}$. This multilayered system provides an additional scenario for examining how the shear 


\section{Chapter 2. Numerical Model}

band formation may be affected by the physical constraint.

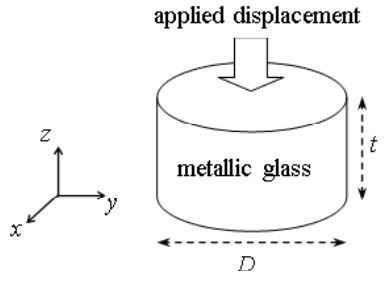

(a)

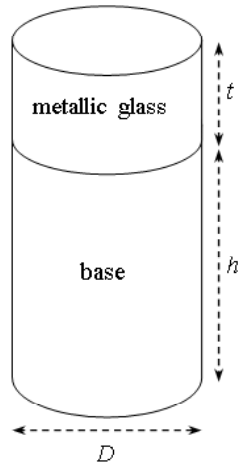

(b)

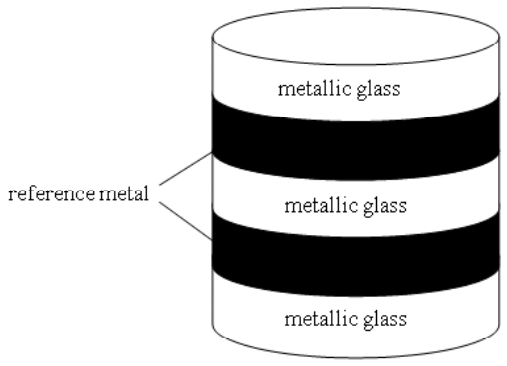

(c)

Figure 2.1: Schematics of the numerical models subjected to compressive loading: (a) a stand-alone metallic glass cyliner, (b) a layer of metallic glass above a supporting base, and (c) multilayered metallic glass/reference metal structure

Perfect bonding between dissimilar materials is assumed in all cases. The total number of elements in the simulations depends on the model type and actual geometry. As an example, in the case of $\mathrm{t}=1 \mu \mathrm{m}$ in Fig. 2.1(b), there are a total of 171,360 eight-noded linear hex elements. The finite element program Sierra/SolidMechanics (Sandia National Laboratories) was used in all calculations under the quasi-static condition. All models and meshes were created with CUBIT Geometry and Mesh Generation toolkit (Sandia National Labs). All metallic materials in the model are treated as isotropic elastic-plastic solids. Young's modulus and Poisson's ratio of the metallic glass were taken to be $118 \mathrm{GPa}$ and 0.37 , respectively. Plastic yielding follows the von Mises criterion and incremental flow theory [29]. The choice of appropriate constitutive laws for amorphous alloys has been a topic of active research. Within the continuum framework, plasticity in crystalline metals is generally controlled only by the deviatoric part of the stress tensor. For disordered materials such as metallic glasses, hydrostatic pressure may be expected to influence the yield 


\section{Chapter 2. Numerical Model}

behavior. Many experimental investigations have concluded that the pressure dependence of plastic deformation is relatively weak (see Ref. [7] for discussion). Some studies specifically showed that the von Mises criterion is adequate for describing the yield response [30,31]. Therefore, for simplicity the von Mises criterion, with perfect plasticity upon yielding at a uniaxial stress of $2.1 \mathrm{GPa}$, is chosen for the present study. It is noted that the plasticity model alone is not able to capture the actual shear banding phenomenon. Rather, in the simulation we incorporated randomly generated weak points in the metallic glass to trigger discrete deformation along the maximum shear directions. The perturbation points, arbitrarily chosen to constitute $1 \%$ of the metallic glass elements, have the same elastic-plastic properties as the regular material elements, except for a built-in work softening response of slope -18.9 GPa upon yielding. Figure 2.2 helps to illustrate the distrubtion of perturbation points in the stand-alone metallic glass model. It is noted that the goal here is not to simulate the actual microscopic processes, but to induce a localized form of plastic flow in the model in a straightforward manner. A banded deformation pattern can be successfully obtained with our current approach. The same numerical methodology has also been employed to elucidate the much improved bending ductility of a surface-coated bulk metallic glass [32].

The Si base in Fig. 2.1(b) is treated as a linear elastic solid, with Young's modulus and Poisson's ratio $107 \mathrm{GPa}$ and 0.172, respectively. An alternative base material was also used; its elastic property is the same as the metallic glass and the yield strength is set at $4.2 \mathrm{GPa}$. Note that this hard metal base is a normal elasticperfectly plastic solid with no mechanism built-in for deformation localization. As for the multilayer model in Fig. 2.1(c), the reference metal is also an elastic-perfectly plastic solid with the same elastic property. Its yield strength, however, is set to be 2.1 GPa which is equivalent to the initial yield point of the metallic glass. Therefore, the multilayer model may be viewed as a single metallic glass cylinder, but with two internal layers divested of the work softening (shear-band forming) capability. In 


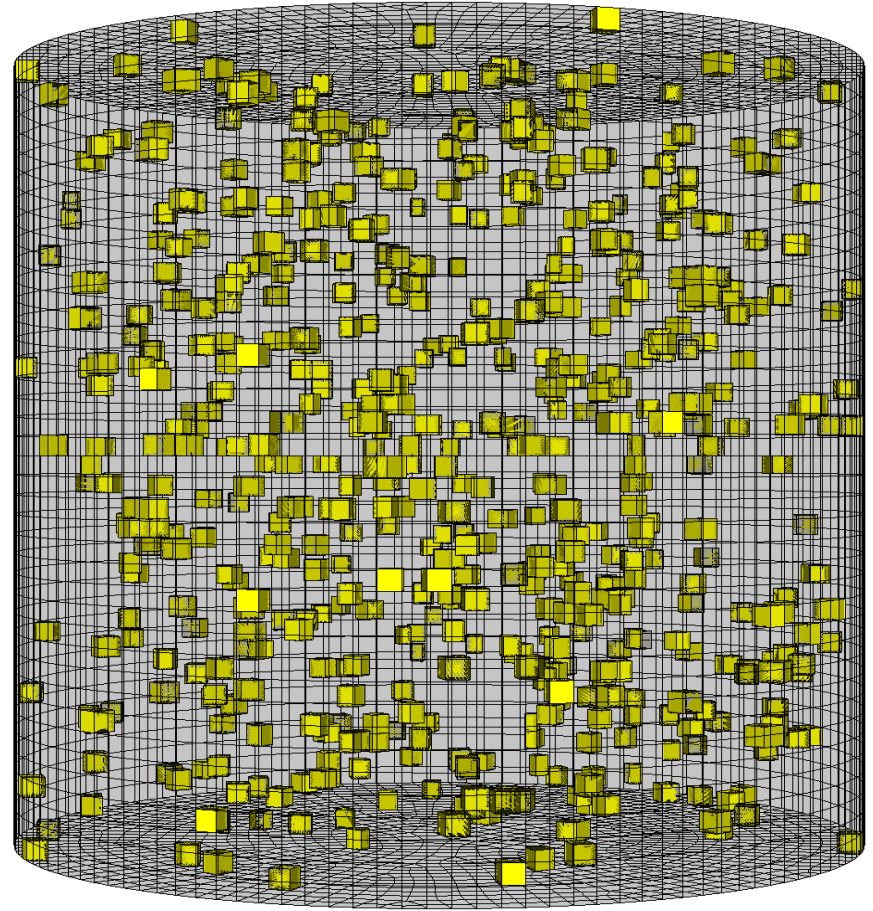

Figure 2.2: Schematic of the distribution of perturbation points in the stand-alone metallic glass model

addition a soft and hard "reference metal" was used in the multilayer case with a yield stress set at 1.05 GPa for the soft metal and a yield stress set at 4.2 GPa for the hard metal.

In the presentation that follows, simulated stress-strain curves of the metallic glass are obtained from the reaction force at each prescribed displacement, on the basis of the engineering stress and engineering strain definitions. In the case of basesupported pillar structure ( Fig. 2.1(b)), the displacement of the base material was first subtracted from the total displacement so the true deformation in the metallic glass can be accounted for. 


\section{Chapter 2. Numerical Model}

\subsection{Beam Model}

A three-diemsional finite element beam model was also constructed. Figure 2.3 shows the schematic of the model consisting of a simple beam. The beam is symmetric about its cross section with a thickness (t) of $2 \mu \mathrm{m}$ and a lengh (l) of $15 \mu \mathrm{m}$. Quasistatic loading is applied using a prescribed pressure on two nodesets placed on the top face (L') 300nm apart and on the bottom face (L) 600nm apart on the beam. Rigid bodies are are created on both faces of the left and right end of the beam. Rigid bodies allow the contrained nodes to rotate while still remaining fixed. The rigid body on the left end of the beam is constrained in all translational directions and the rotations are fixed in the $\mathrm{x}$ and $\mathrm{z}$-directions. The rigid body on the right end of the beam is constrained in the $\mathrm{y}$ and $\mathrm{z}$-directions with roatations about the $\mathrm{x}$ and z-directions fixed. The beam is constructed as an isotropic elastic-plastic metallic material. Young's modulus and Poisson's ratio of the metallic glass were again taken to be $118 \mathrm{GPa}$ and 0.37 . Perturbation points that constitute $1 \%$ of the metallic glass elements are added to the beam model in order to facilitate plastic flow in the model. The beam mesh consisted of 61,600 eight-noded linear hex elements.

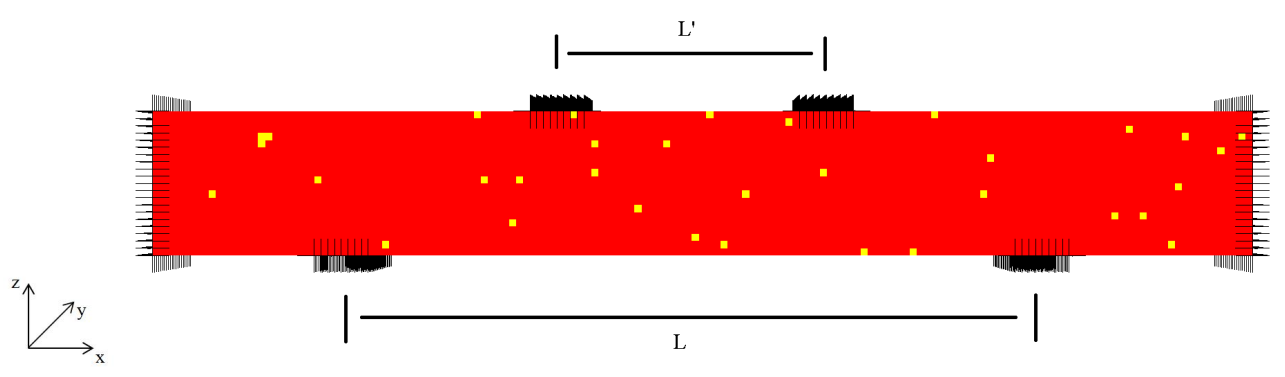

Figure 2.3: Schematic of the numerical beam model subjected to four point bending. 


\section{Chapter 3}

\section{Mesh Convergence Study}

\subsection{Background}

Mesh convergence along with validation is of great importance when performing finite element computer simulations. To enure that the solution obtained is as close to reality as possible, solutions should be obtained from multiple meshes starting with a "coarse mesh" and ending with a "fine mesh". However the terms "coarse" and "fine" are relative. A coarse mesh is the first mesh to be run that is adequate to solve the problem. After the coarse mesh is run a second mesh is generated that consists of a greater number of elements. If the difference in solution between the first and second mesh is large further refinements are performed until the difference in solution is negligible. Mesh refinement studies are almost always used when running any accurate finite element analysis and certain standards have been established. A mesh refinement should meet the following criteria [33]:

- All previous meshes should be contained in the refined mesh

- Every point of the domain can be included within an arbitrarily small element 


\section{Chapter 3. Mesh Convergence Study}

at any stage of the mesh refinement

- The same order of approximation for the solution may be retained through all stages of the refinement process.

Although a mesh refinment study is the most precise way of creating an adequate discretization that will reach a converged solution it is not always feasible in practice due to the computational cost involved. When computational cost becomes a primary concern the finite element modeler must use one's judgement to discern whether or not a mesh can be considered good quality. A good quality mesh is often dictated by the geoemtry and qualitative understanding of the variables of the solution. The descision when to stop further refinement should be reached by scientific knowledge and experience with a given class of problems [33].

\subsection{Numerical Model}

A mesh convergence study was performed on the stand-alone metallic glass model shown in Figure 2.1(a) in order to show the type of discretization that is needed for the analysis to reach a converged solution. The largest aspect ratio $\left(t_{f} / D=1\right)$ metallic glass cylinder was used for this study. Quasi-static compressive loading is applied via a prescribed displacement on the top face of the model. The bottom face of the model is fixed in the z-direction while the $\mathrm{x}$ and $\mathrm{y}$ directions are not constrained. The h-method of mesh refinement was used for this study in which every element through out the mesh is refined during each level of remesh. Traditional methods of uniformed mesh refinement dictate that each hex element of the model is split in two directions therefore turning a single hex element into eight hex elements for each level of refinement. Figure 3.1 shows an example of how the uniformed mesh refinement was carried out for each hex element. 


\section{Chapter 3. Mesh Convergence Study}
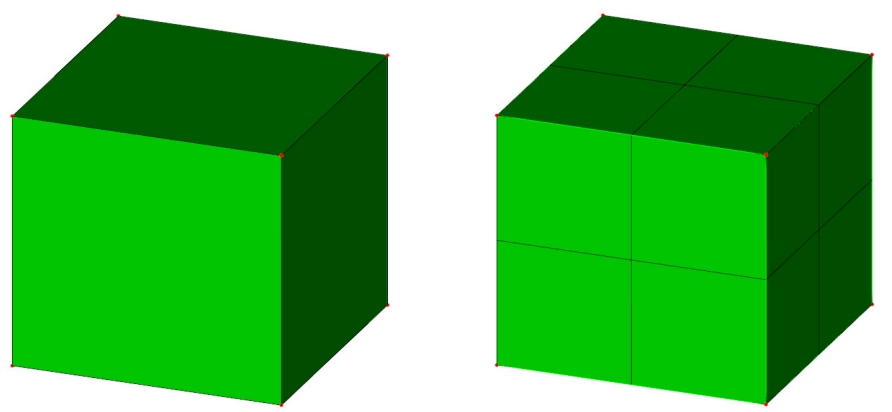

Figure 3.1: Uniform mesh refinement of single hex element

A total of five models consisitng of four levels of refinement were used in this convergence study and can be seen in Figure 3.2. In the figure the elastic-plastic metallic glass elements are red and the perturbation points with built in softening response are yellow. The pertubation points represent $1 \%$ of the total elements in each model with the distribution of the points being random for each mesh. A coarse mesh Fig 3.2(a) consisting of only 364 hex elements was initally used to establish a base line that was deemed adequte to solve the probelm. Ultimatley a metallic glass model Fig 3.2(e) consisting of approximately 1.5 million elements was ran before a converged solution was reached.

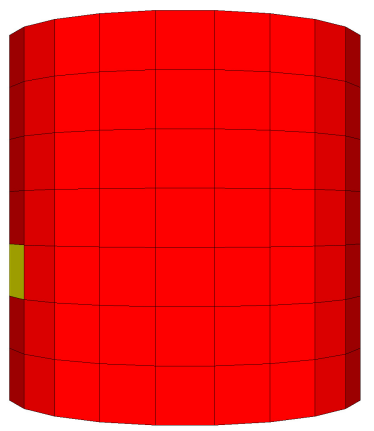

(a)

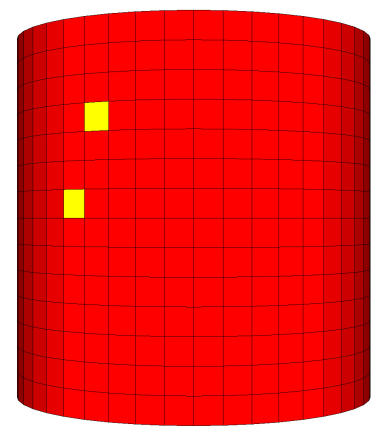

(b) 
Chapter 3. Mesh Convergence Study

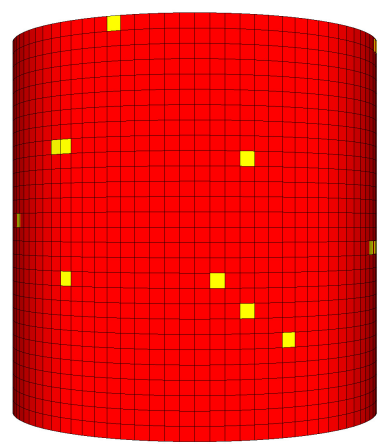

(c)

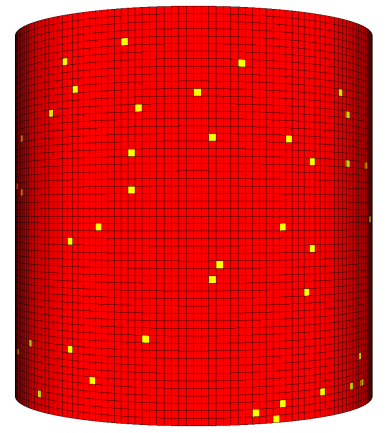

(d)

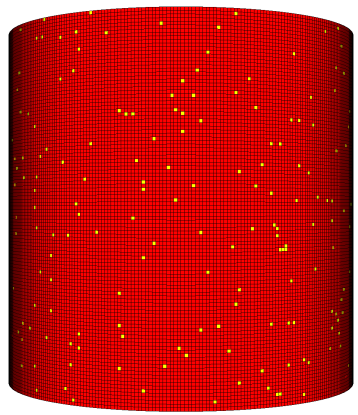

(e)

Figure 3.2: Stand-alone metallic glass mesh refinements: (a) mesh 1, (b) mesh 2, (c) mesh 3, (d) mesh 4, (e) mesh 5

\section{$3.3 \quad$ Results}

Figure 3.3 shows a magnifed plot of the engineering stress vs engineering strain and one can see that the stress is increasing as a result of mesh refinement. The stress level jumps significantly from the orginal coarse mesh to the first level of mesh refinement which suggests the need for further refinement. Again there is a varaiance in solution between mesh 2 and mesh 3 however it is not quite as severe as the change from the mesh 1 to mesh 2 . The stress continues to rise with further mesh refinement untill the difference in solution between meshes 4 and 5 is small enough that another level of refinement would not produce a large disparity in solution from the previous 
mesh.

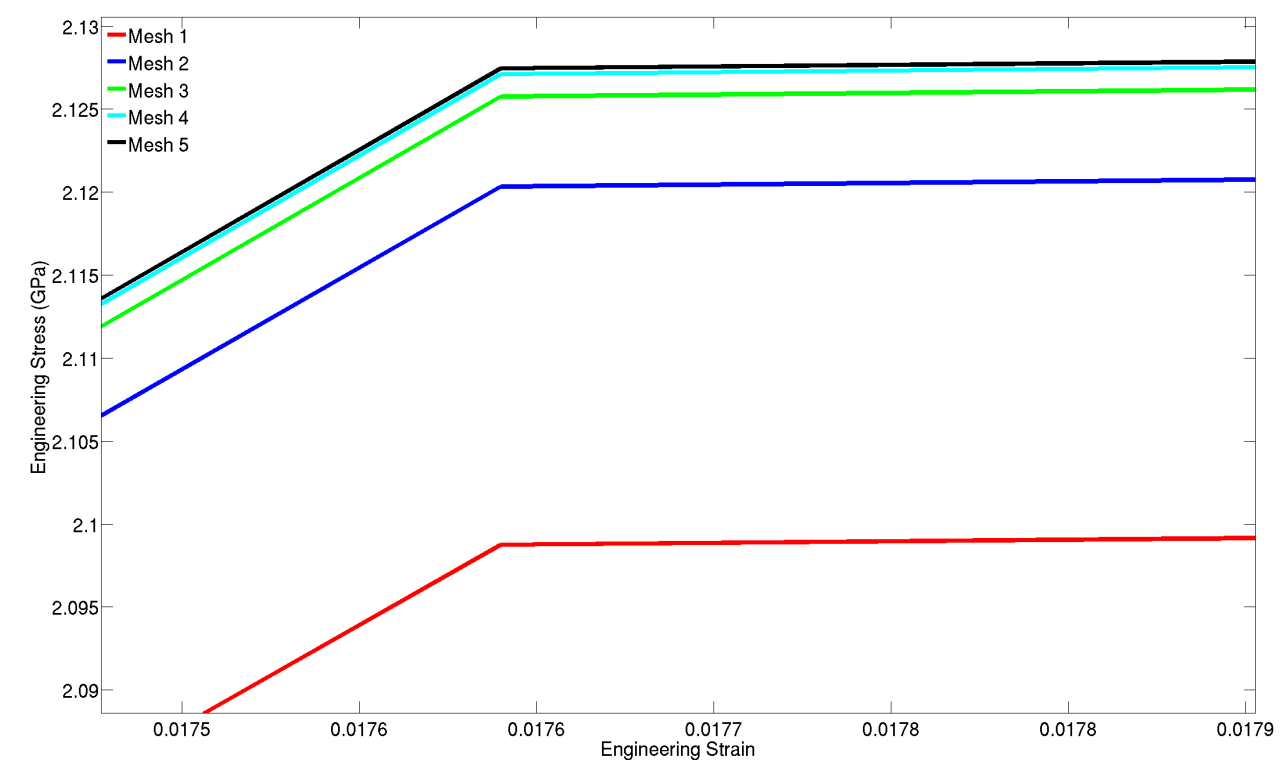

Figure 3.3: Stand-alone metallic glass mesh refinement - Stress vs. Strain

Figure 3.4 shows a more convential log scale plot used to verify convergence in which the max yield stress serves as the analytic value used to verify convergence. The max yield stress is taken at a strain offset of $10 \%$ and is plotted against the element count of each model. From the plot, one can see that as the solution approaches the converged magnitude the slope of successive line segements approaches zero. Clearly the problem is reaching a converged solution between mesh iterations 4 and 5. In most cases, if analytic value used to measure convergence is changing by less than $10 \%$ from one run to the next, there will not be much benefit from pushing the mesh refinement any further [34].

Table 3.1 offers more information on the amount of elements contained in each model, the number of processors used and the runtime for each model. Note the run time listed in the table is the total run time for the test and not the indiviudal 
Chapter 3. Mesh Convergence Study

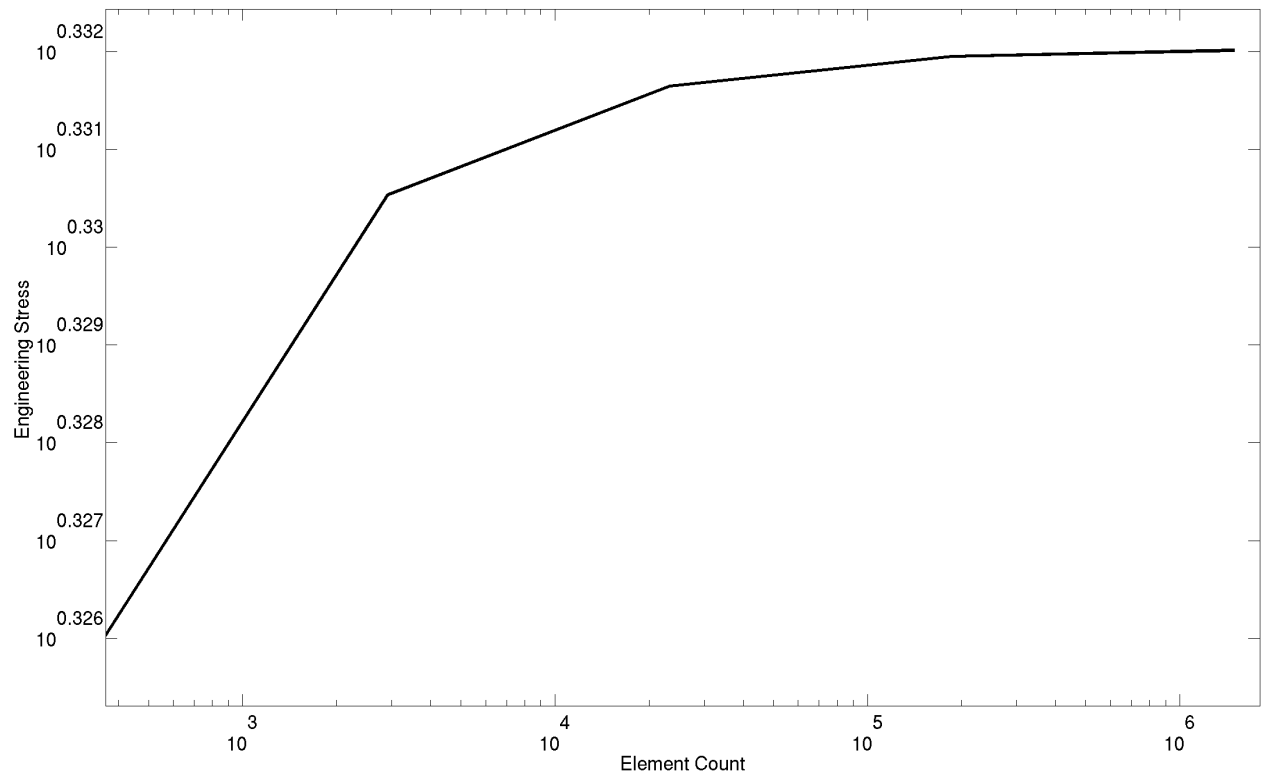

Figure 3.4: Yield stress for various levels of mesh refinement

processor run time. The table shows that the analysis begins to get computationally exspensive starting with mesh 4 being run on 8 processors for an hour. Mesh 5 is the most computationally expsenive model running on a total of 80 processors for a total run time of approx. 28 hours. All models used for this mesh refinement study were run on Sandia National Laboratories super computer designated Redsky.

\begin{tabular}{|l|l|l|l|l|l|}
\cline { 2 - 6 } \multicolumn{1}{c|}{} & mesh 1 & mesh 2 & mesh 3 & mesh 4 & mesh 5 \\
\hline Processor Count & 1 & 1 & 2 & 8 & 80 \\
\hline Number of Elements & 364 & 2,912 & 23,296 & 186,368 & $1,490,944$ \\
\hline Total Run Time & 2secs & 18 secs & 6 mins 43secs & 1hr 43mins & 28hrs 19mins \\
\hline
\end{tabular}

Table 3.1: Stand-alone metallic glass parameters for mesh convergence study. 
Chapter 3. Mesh Convergence Study

\subsection{Conclusion}

It was found that in order to reach a converged soultion a mesh consisting of approximatly 1.5 million elements would need to be used. A mesh of that size is extremely exspensive to not only mesh but to also run and is completely unfeasable without the use of a high perfromance supercomputer. Table 3.1 highlights how computationally exspensive some of the meshes were to run. With the most refined mesh taking approximatly 28 hours to run it would not be feasible or cost effectve to use such an accuarte model. In order to help keep the computational cost low a mesh consisting of $60 \mathrm{~K}$ elements was used for the proceeding stand-alone metallic glass models. 


\section{Chapter 4}

\section{Results and Discussion}

\subsection{Stand-alone Metallic Glass Cylinder}

Numerical results based on the model in Fig. 2.1(a) are first presented. Figure 4.1 shows the simulated stress-strain curves of the metallic glass cylinders with various aspect ratios (defined to be t/D). It can be seen that, under uniaxial loading free of external constraint, the same stress-strain curves are obtained regardless of the geometry. Figures 4.2(a) and 4.2(b) show the contour plots of equivalent plastic strain in the specimens with aspect ratios 1.0 and 0.6, respectively, when the overall compressive strain is 0.042 . It is noted that the actual displacment of the metallic glass is not shown in the contour plots. The localized deformation pattern is evident. Shear bands appear to be uniformly distributed throughout the material, and the plastic strains inside the bands are much greater than the surrounding. The extent of shear bands, in terms of both density and maximum plastic strain, for the two aspect ratios appears to be the same, which is consistent with the equivalence of overall stress-strain behavior observed in Fig. 4.1. 
Chapter 4. Results and Discussion

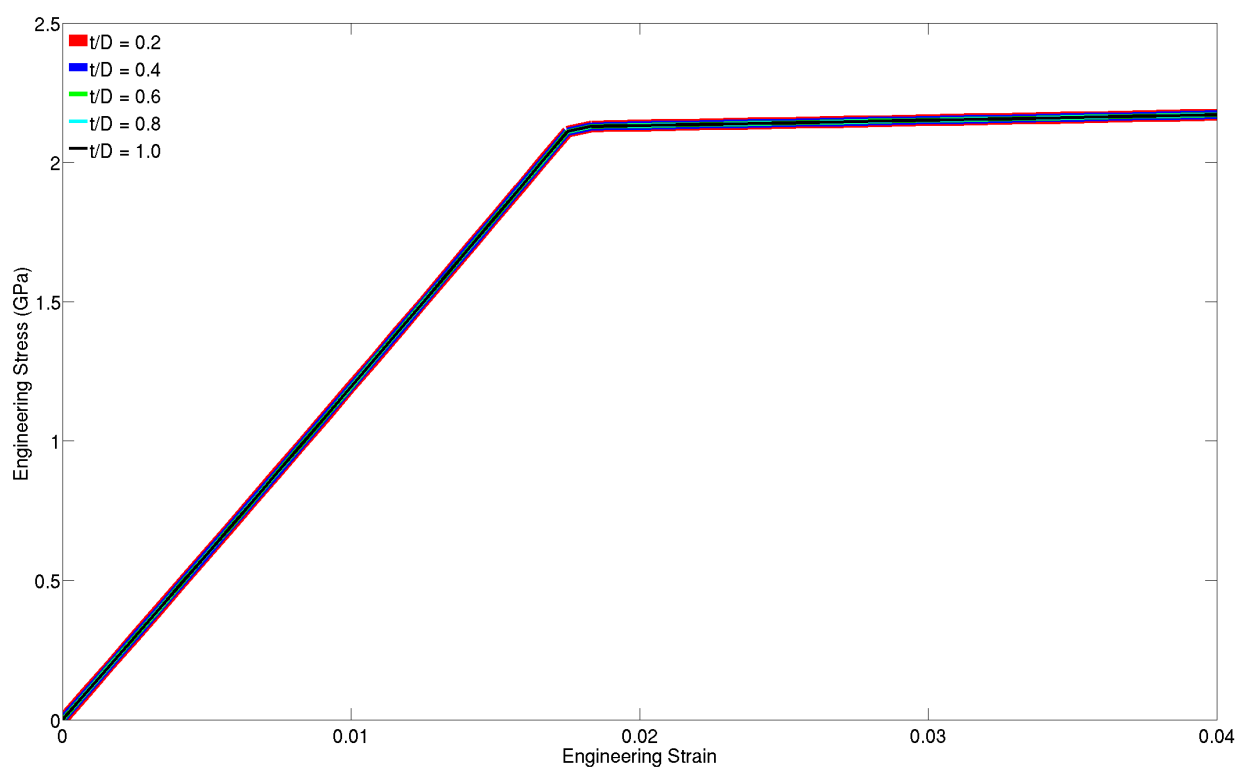

Figure 4.1: Simulated stress-strain curves for the stand-alone metallic glass cyliners with aspect ratios $0.2,0.4,0.6,0.8$ and 1.0

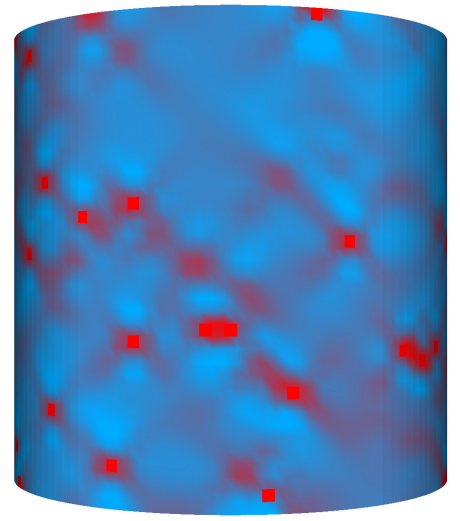

(a)

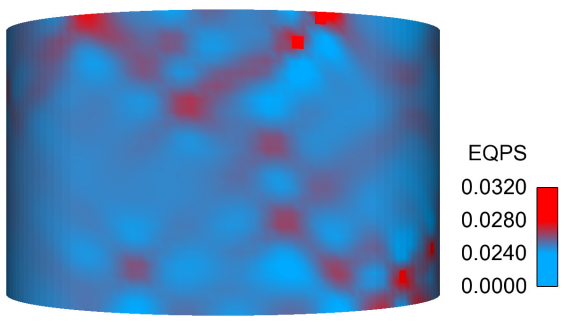

(b)

Figure 4.2: Contours of equivalent plastic strain in the stand-alone metallic glass cylinders with aspect ratio (a) 1.0 and (b) 0.6, at the same macroscopic strain of 0.042 . 


\subsection{Cylinders Constrained by Base Material}

The substrate-supported pillar model in Fig. 2.1(b) is now considered. Figure 4.3 shows the simulated stress-strain curves of the metallic glass cylinders when they are attached to a $\mathrm{Si}$ base. It is observed that the different aspect ratios result in essentially the same elastic behavior. However, the plastic flow stress increases with decreasing aspect ratio. In other words, when the bottom face of the metallic glass is constrained by an elastic substrate, a shorter cylinder (becoming disk-like) will display a higher apparent mechanical strength. This is due to the decreasing volume that is relatively free to facilitate uninterrupted shear path along the 45 directions, as seen below.

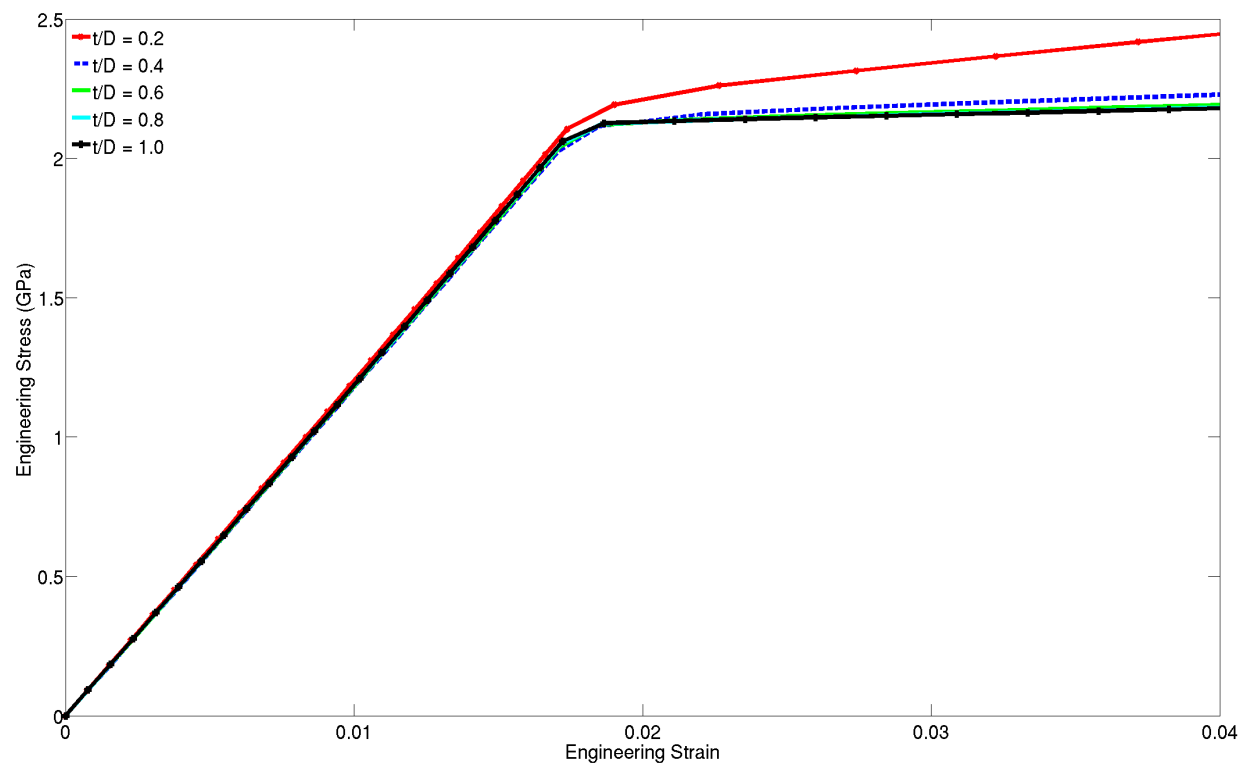

Figure 4.3: Simulated stress-strain curves for the metallic glass cyliners, with aspect ratios $0.2,0.4,0.6,0.8$ and 1.0 , when they are attached to a Si base.

Figure 4.4 shows the yield stress as a function of aspect ratio of the metallic glass. Here the yield stress is defined to be the plastic flow stress at $1 \%$ offset strain. Note 


\section{Chapter 4. Results and Discussion}

that this curve shows the same trend as in the pillar compression experiment $[2,17]$. When the aspect ratio is greater than about 0.5 , the yield stress stays nearly constant. Below 0.5 a steep increase in yield stress is seen. Figures 4.5(a) and 4.5(b) show the contour plots of equivalent plastic strain when the overall compressive strain of the metallic glass portion is at 0.042 , in the models with aspect ratios of 1.0 and 0.6 , respectively. It is notable that shear bands are more populated near the top of the specimen, away from the interface with Si. Plasticity is also much stronger in Fig. 4.5(a). Apparently the interfacial constraint, causing higher magnitudes of hydrostatic stress locally, tends to suppress plastic deformation in the metallic glass. Such an influence is thus greater in low-aspect-ratio models.

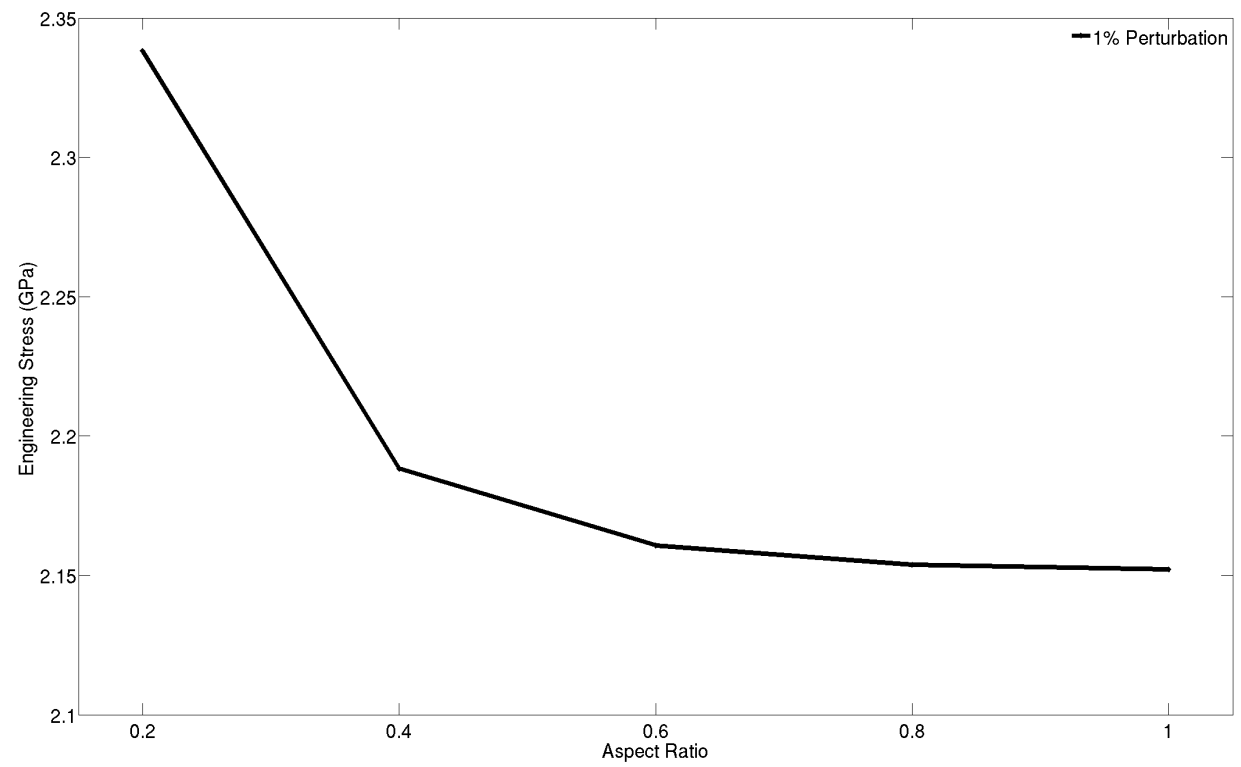

Figure 4.4: Simulated 1\% offset yield stress as a function of the aspect ratio of the Si-attached metallic glass.

To examine the possibly different effects of interfacial constraint on localized deformation as opposed to homogeneous deformation (traditional metal plasticity), we undertook a separate set of simulations using the same model configuration ( Fig. 


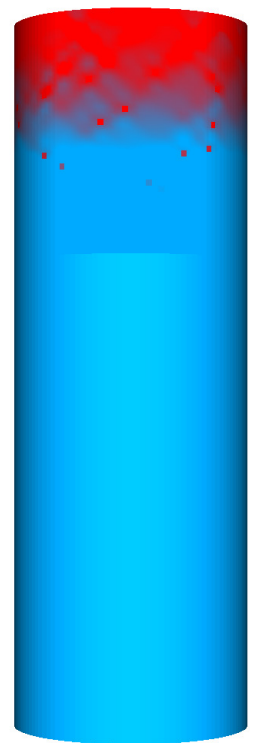

(a)

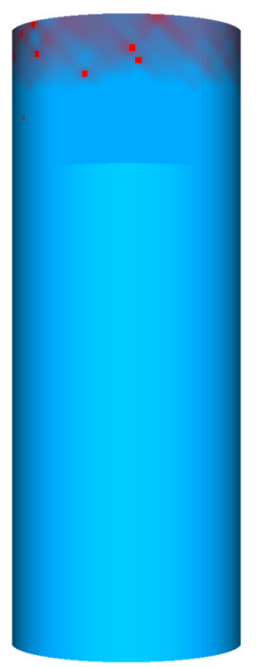

EQPS

0.0380

0.0340

0.0220

0.0000

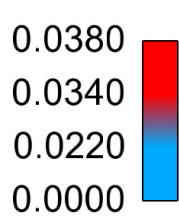

(b)

Figure 4.5: Contours of equivalent plastic strain in the Si base-attached metallic glass cylinders with aspect ratio (a) 1.0 and (b) 0.6, at the same macroscopic strain of 0.042 .

2.1(b)) but without the perturbation points that trigger shear bands. This means that the metallic glass under this special circumstance is simply an elastic-perfectly plastic material with the same elastic property and initial yield strength. Furthermore, we also included a case where an excessive number of perturbation points, namely $10 \%$ of the material elements, are incorporated into the metallic glass. It was found that these two additional cases lead to stress-strain curves similar to those in Fig. 4.3 (not shown here). When the simulated yield stress is plotted against the aspect ratio, Fig. 4.6, the same trend as in Fig. 4.4 is observed. The lower yield stress for the higher proportion of perturbation points is a consequence of more prominent work softening, while the overall trend with the aspect ratio remains unaffected. It is thus realized that the Si base-induced constraint influences the apparent strength of the metal cylinder in fundamentally the same way, irrespective of the localized or 


\section{Chapter 4. Results and Discussion}

homogeneous form of deformation. It is worth mentioning that, in traditional metal plasticity, constrained deformation is also dictated by the ease of flow along the 450 shear path $[1,35,36]$.

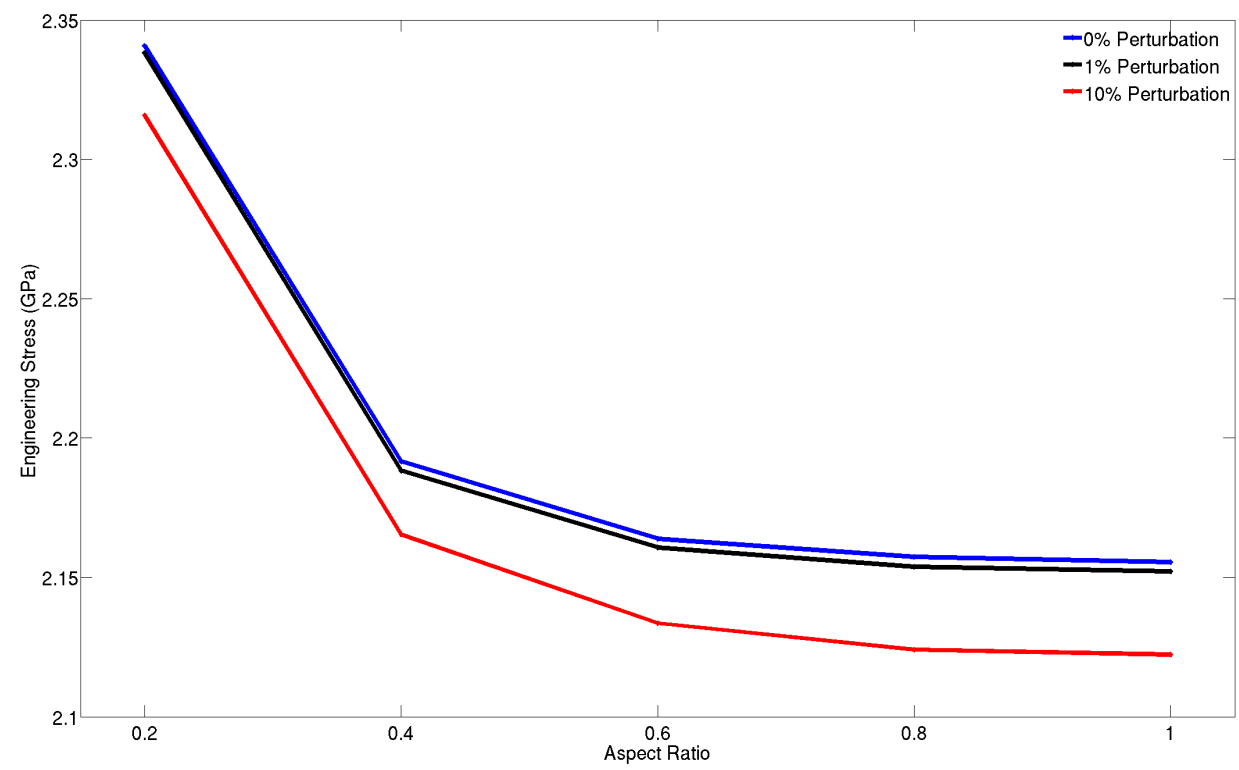

Figure 4.6: Simulated 1\% offset yield stress as a function of the aspect ratio of the Si-attached metallic glass. In addition to the standard model of $1 \%$ perturbation points, two additional cases, with 0 and $10 \%$ perturbation points, are included.

Figures 4.7(a) and 4.7(b) show the contour plots of equivalent plastic strain, in the case of an elastic-perfectly plastic metallic glass (no perturbation point) of aspect ratios of 1.0 and 0.6 , respectively, when the macroscopic compressive strain of the metal portion is at 0.042 . Although the material is capable of homogenous deformation, the interfacial constraint renders higher plastic strains near the top regions, especially in the higher-aspect-ratio model. Note that this observation has direct implications in interpreting experimental results of pillar compression for substratebonded crystalline metals. In the case of metallic glasses, the deformation pattern is manifested by the denser shear bands in the upper region and in the higher-aspect- 
ratio specimens (Fig. 4.5).

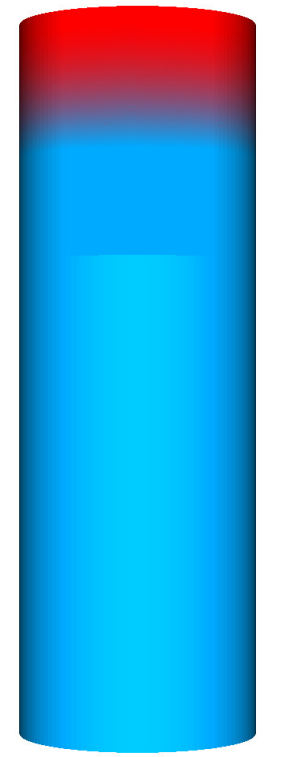

(a)

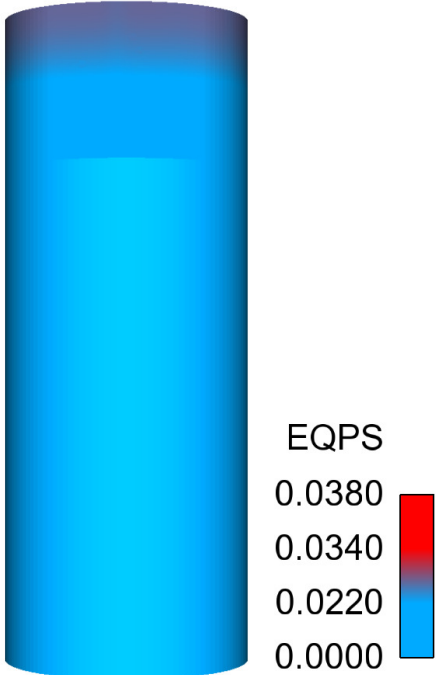

(b)

Figure 4.7: Contours of equivalent plastic strain in the Si base-attached metallic glass free of any perturbation points, with aspect ratios (a) 1.0 and (b) 0.6 at the same macroscopic strain of 0.042 .

In addition to the Si base, a hard metal base is also employed in this study. The hard metal is an elastic-perfectly plastic solid with a yield strength of $4200 \mathrm{MPa}$ (much greater than the initial yield strength of the metallic glass). The standard constitutive model for the metallic glass described in Section 2 is used here. We seek to assess how the shear banding configuration in the metallic glass can be affected by a plastically deforming support structure. The result is presented in Figs. 4.8(a) and 4.8(b) where the contour plots of equivalent plastic strain corresponding to aspect ratios of 1.0 and 0.6, respectively, under the macroscopic compressive strain of 0.042 in the metallic glass portion, are shown. It can be seen, in comparison with the $\mathrm{Si}$ base in Fig. 4.5 under the same overall strain, that a hard but ductile base can allow slightly more shear bands to develop in the metallic glass. Shear bands are still more 
populated in the upper region of the specimen. Since the base material also has the ability to plastically deform, the interface becomes less discernible in Fig. 4.8 compared to the case of an elastic Si base in Fig. 4.5.

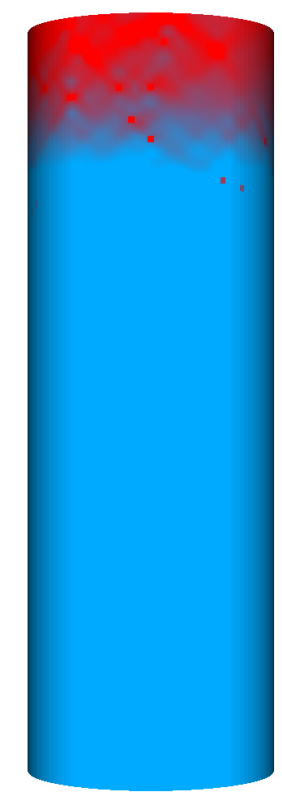

(a)

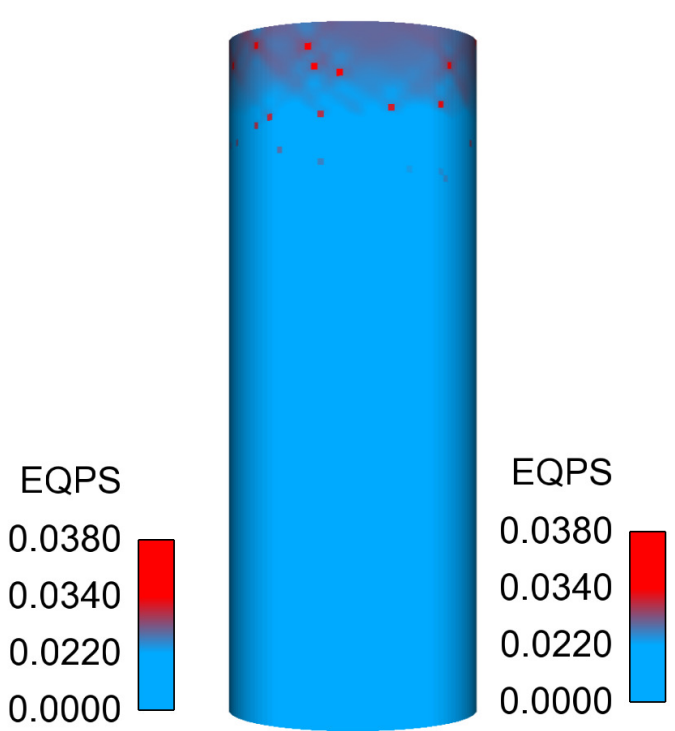

(b)

Figure 4.8: Contours of equivalent plastic strain in the "hard metal" base-attached metallic glass with aspect ratios (a) 1.0 and (b) 0.6 at the same macroscopic strain of 0.042 .

To this end, it is noted that a ductile base with a yield strength lower than that of the metallic glass was also included in our preliminary study. Under this circumstance, plasticity mainly occurs in the base material rather than the metallic glass so the result is not presented here.

\subsubsection{Comparison with Experimentation}

This section compares the numerical results presented above with some recent experimental work done on TFMGs. Current research on TFMGs has focused on under- 


\section{Chapter 4. Results and Discussion}

standing the mechanical properties of TFMGs via systematic studies. This thesis was designed to be simliar to experimental work being performed by researchers around the world $[2,19,21,23]$. A recent paper published by Ye et al. [2] looked at the properties of a Zr-based TFMG on silicon substrate. Their study consisted of subjecting composite micropillar specimans to compression tests. The micropillars were created by depositing a TFMG of varying thickness (400, 600, 800 and 1000nm) on top of an Si substrate. The micropillars are then milled out of the TFMG/Si composite using a focused-ion-beam. It is noted that due to ion-beam divergence the FIBed micropillars have a slight taper (approx. 3degrees) and its effect on the numerical experiment performed in this thesis will be investigated later in the results. Figure 4.9 shows an FEM micrograph of the milled micropillars used in the experimental research.

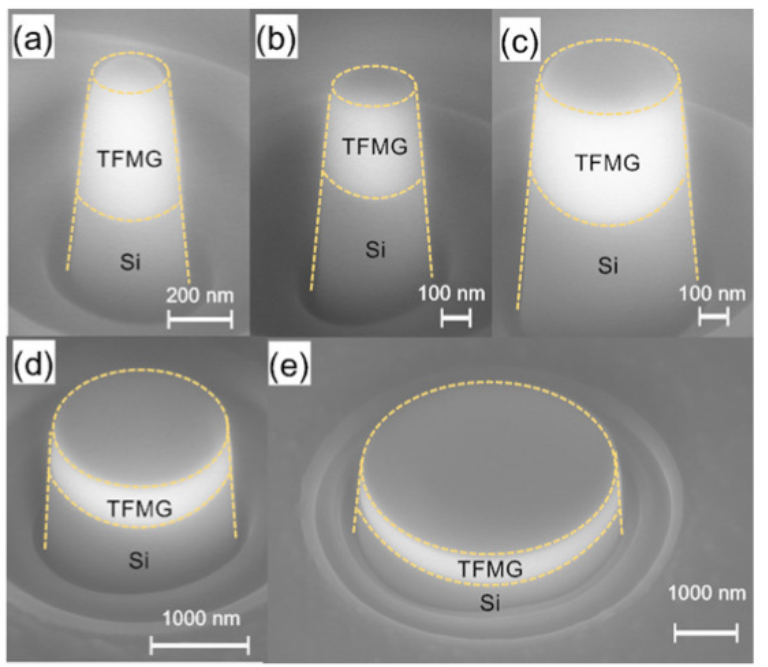

Figure 4.9: (a)-(e) SEM micrographs showing the TFMG/Si composite micropillars with different sizes and shapes. Note that a sketched profile of the micropillar is superimposed on the corresponding SEM micrograph for clarity. [2]

As mentioned earlier the microcompression experiment performed on the thin film metallic glass-silicon micropillar produced very similar results to the numerical sim- 


\section{Chapter 4. Results and Discussion}

ulation just disscused. Figure 4.10 shows the yield stress of the TFMG's calculated experimentally as a function of the aspect ratio. Comparing the numerical results in Fig. 4.4 with the experimental results in Fig. 4.10 one can see that the same trend is produced. This trend helps highlight the size effect often found in TFMGs and how it can affect yield stress and other mechanical properties in amorphous metals.

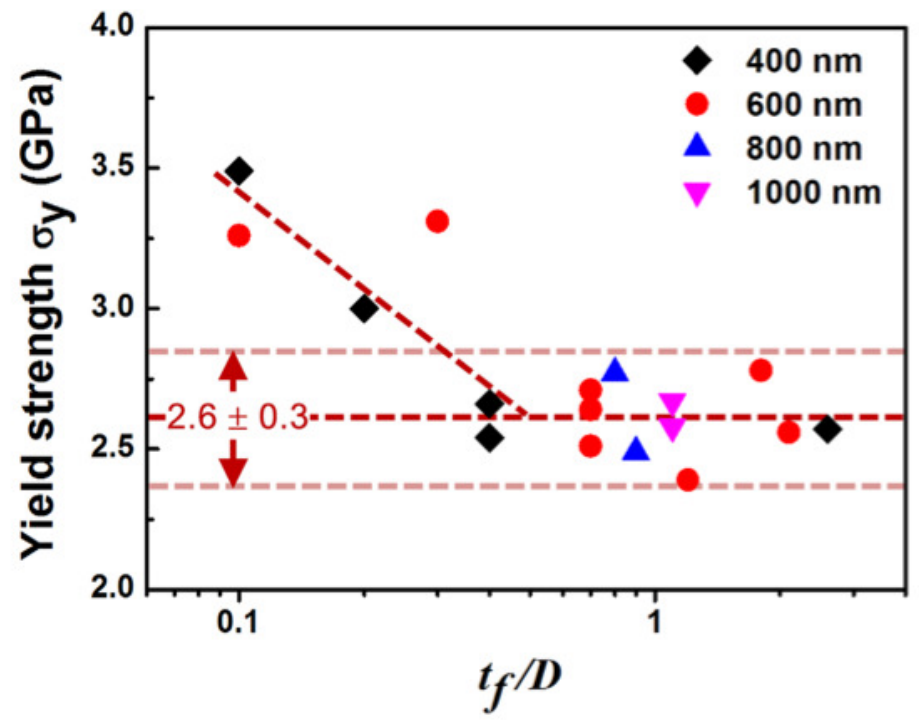

Figure 4.10: The variation of the yield strengths of the TFMGs obtained from microcompression with their $t_{f} / D$ ratios. $[2,17]$

\subsection{Multilayered Structure}

Attention is now turned to the multilayer model in Fig. 2.1(c). The reference metal sandwiched between the metallic glass layers is elastic-perfectly plastic, with the yield strength equal to the initial yield strength of the metallic glass. Figure 4.11 shows the simulated stress-strain curves of the layered model and the all-metallic glass model of the same dimension (i.e., the curve of $t / D=1$ in Fig. 4.1). The two 
Chapter 4. Results and Discussion

curves essentially coincide.

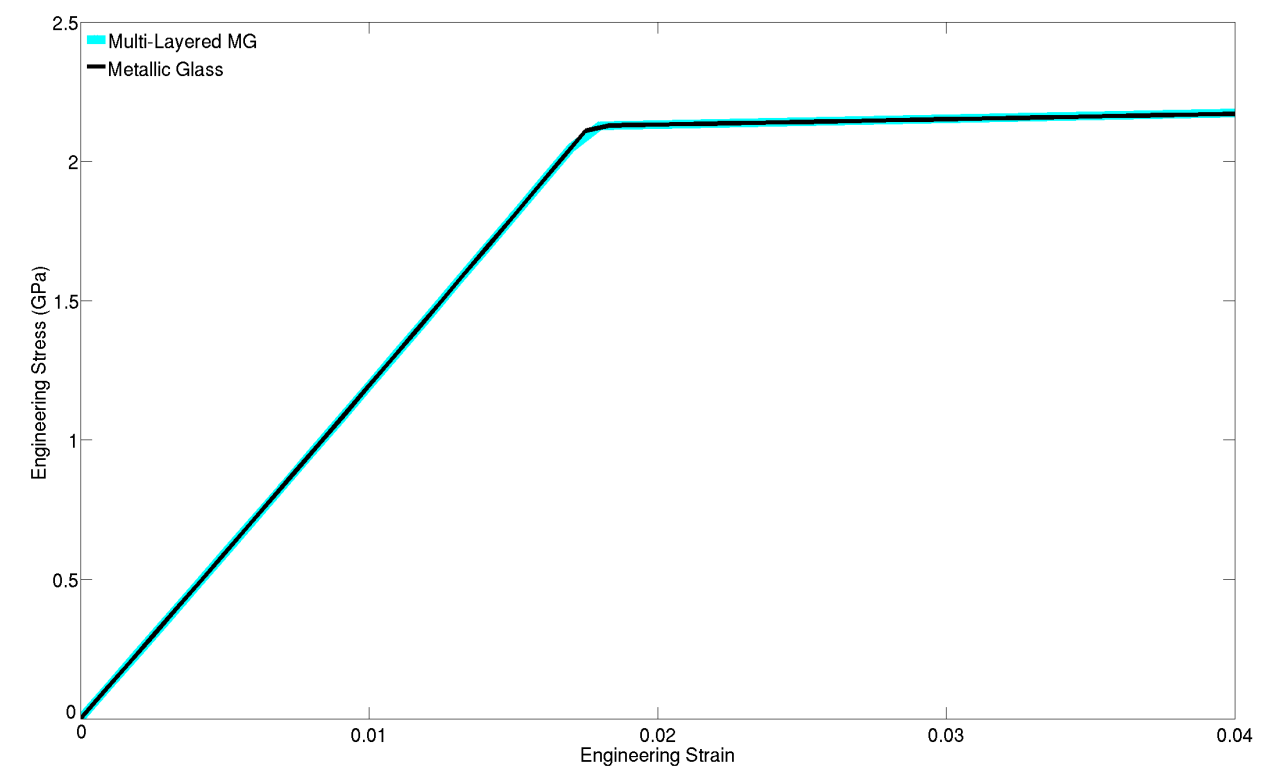

Figure 4.11: Simulated stress-strain curve of the multilayer model. Also included is the stand-alone metallic glass model of the same overall dimension (the case of $\mathrm{t} / \mathrm{D}=1$ in Fig. 2).

Figure 4.12 shows the contour plot of equivalent plastic strain in the multilayer model, when the overall applied compressive strain is 0.042 . While there is no perturbation point inside the reference metal, extension of shear bands from the adjacent metallic glass layers into the reference layers has occurred. By comparing Fig. 4.12 with Fig. 4.2(a), the insertion of reference metal layers is seen to cause discontinuity of localized deformation paths at the interfaces, and the overall shear band population and plastic heterogeneity are slightly reduced. It is anticipated that, if strain hardening capability is built into the reference metal or if a compliant interface exists in the model, localization of plasticity will be further reduced.

Several models were constructed with the "reference metal" having a built in hardening response of slope $2.1 \mathrm{GPa}$ (10\% Strain Hardening), $5.25 \mathrm{GPa}$ (25\% strain 
Chapter 4. Results and Discussion

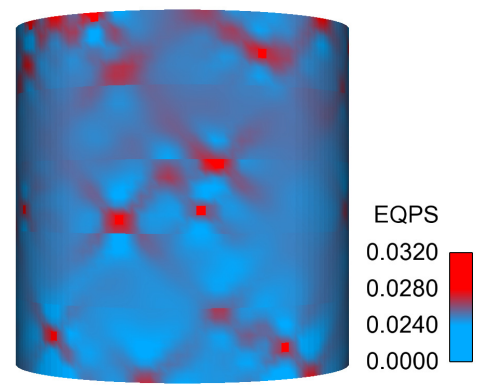

Figure 4.12: Contour plot of equivalent plastic strain in the multilayer model when the macroscopic strain is at 0.042 .

hardening), $10.5 \mathrm{GPa}$ (50\% strain hardening) and $21 \mathrm{GPa}$ (100\% strain hardening) upon yielding. Figure 4.13 shows the equivalent plastic strain contour plot for the multilayer models with added hardening response of $10 \%$ and $25 \%$ strain hardening at a compressive strain of 0.042. Comparing Fig. 4.13(a) and Fig. 4.13(b) with Fig. 4.12 one can see that the localization of plasticity is being reduced with the added hardening and shear banding is almost non-existent in the reference metal containg $25 \%$ strain hardening. To examine how the shear bands interact with the referance material as more strain hardening is added the EQPS scales had to be adjusted. Figure 4.14 shows all the hardedning models with adjusted and varying EQPS scales.

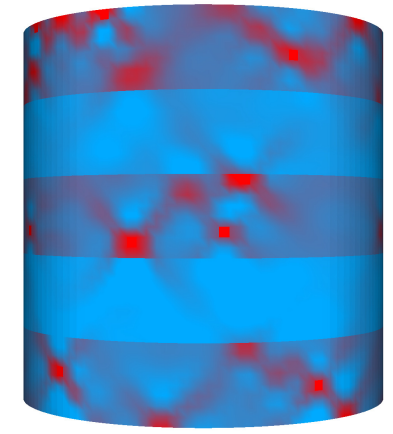

(a)

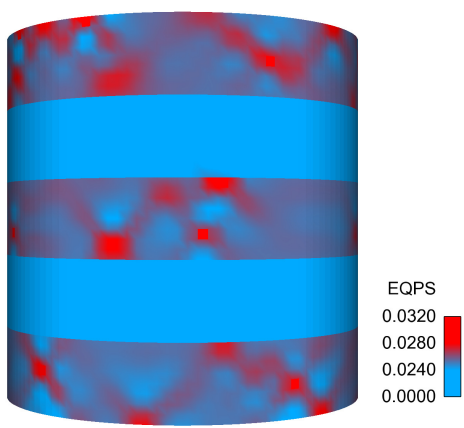

(b)

Figure 4.13: Multilayer metallic glass model with built in hardening response: (a) 10\% Strain Hardening, (b) 25\% Strain Hardening 
Chapter 4. Results and Discussion

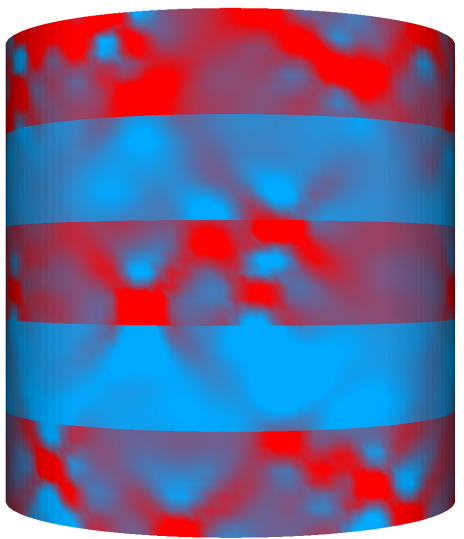

(a)

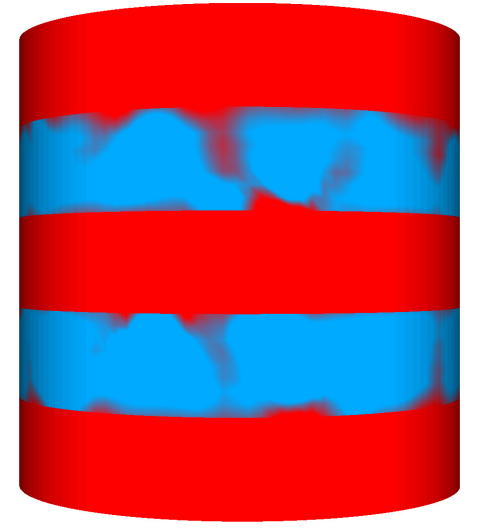

(c)

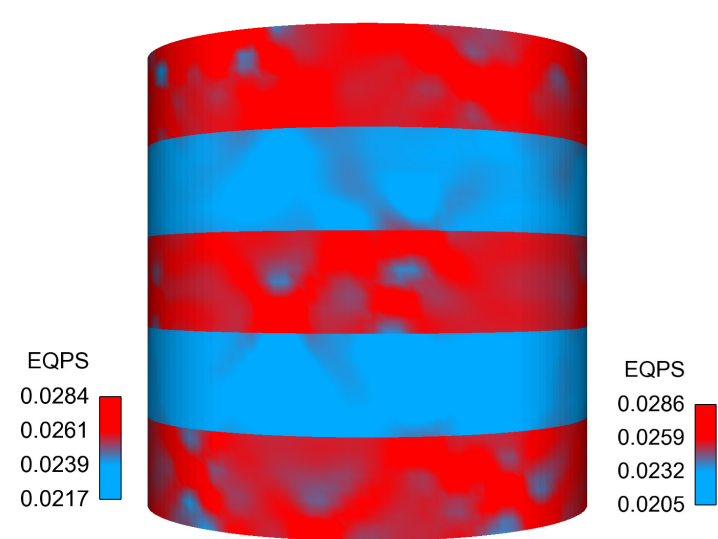

(b)

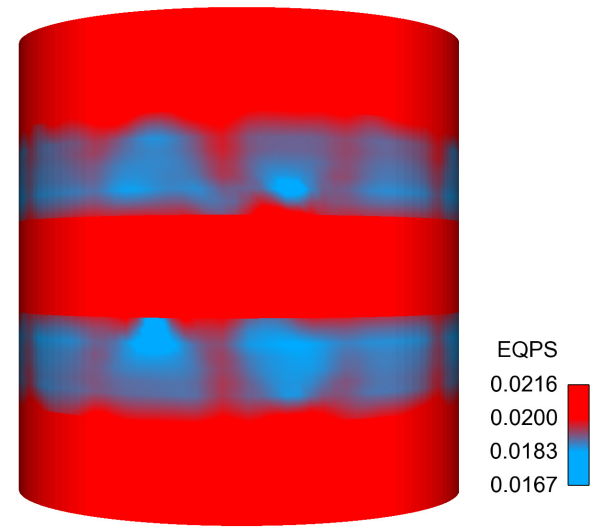

(d)

Figure 4.14: Multilayer metallic glass models with built in hardening response. The EQPS scale has been adjusted for each model to better show the development of shear bands. (a) 10\% Strain Hardening, (b) 25\% Strain Hardening, (c)75\% Strain Hardeding and (d) 100\% Strain Hardening 


\section{Chapter 4. Results and Discussion}

Another multilayer model was created with the reference metals having different hardening responses with the top reference metal having the $10 \%$ hardening response and the bottom reference metal having the $50 \%$ hardening response. Figure 4.15 shows the equivalent plastic strain contour plot for the multilayer model containing the reference materials with different hardening responses. At a compressive strain of 0.042 the plastic deformation is contained primarily within the first three layers of the model with only the perturbation beginning to yeild in the bottom two layers. The $50 \%$ strain harden metal keeps the shear bands from propagating further through the model. As anticipated the strain hardening of the reference metal plays a big role in the evolution of shear bands in metallic glass.

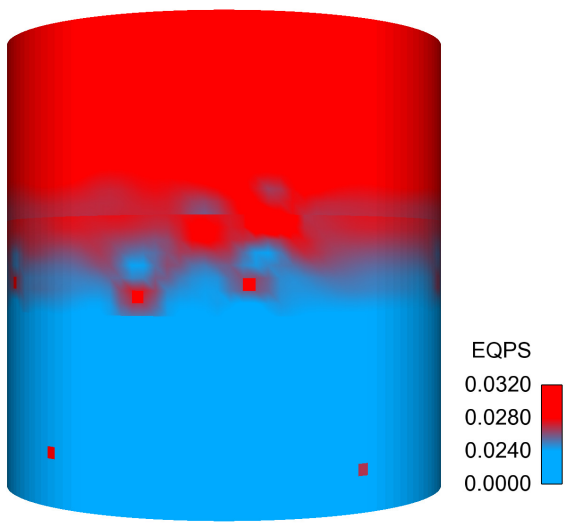

Figure 4.15: Contour plot of equivalent plastic strain in the multilayer model when the macroscopic strain is at 0.042 .

\subsubsection{Comparison with Experimentation}

It is worth mentioning that multilayered pillars consisting of alternating metallic glass and other metal layers are also an active area of experimental research [37-39]. One of the inherit limitations of metallic glasses in both bulk and thin film have been the materials brittleness. Current research looks at adding a thin layer of 


\section{Chapter 4. Results and Discussion}

nanocrystalline metal film beneath the brittle metallic glass film. The underlying nanocrystalline metal serves two purposes: one is to produce the unqiue inelastic shear slip transfer at the amporphous-crystal interface and the other is to suppress local stress concentrations due to dislocation pileups [37]. Again micropillar beams are constructed on $\mathrm{Si}$ wafers consisting of alternating layers of amorphous-crystal layers and are subject to compressive loading. Figure 4.16 shows an SEM micrograph of a multilayer micropillar subjected to compression. The recent experimetal research showed that shear bands initiated in the metallic glass films were found to be accommodated at the interface with the adjacent layer, which resulted in overall more homogeneous deformation and thus much improved ductility compared to monolithic metallic glass structures. The experimental findings are in qualitative agreement with the present discussion.

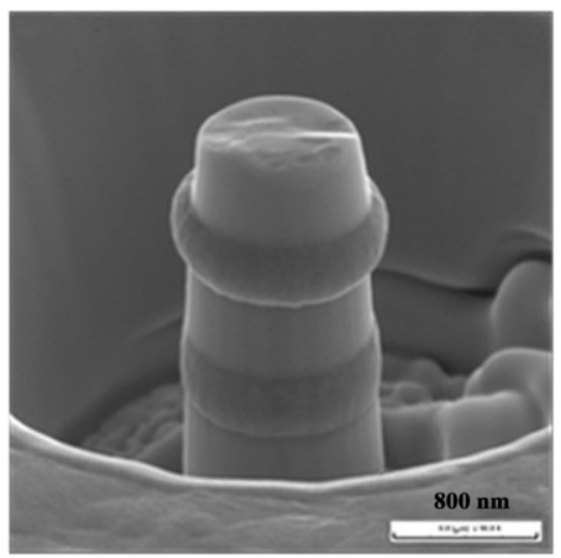

Figure 4.16: SEM micrograph showing $\mathrm{ZrCu} / \mathrm{Cu}$ five-layer pillar compressed to a predetermined displacment of 300nm [37] 


\subsection{Effect of Taper}

In experiments, a slight taper is frequently seen in the FIB (focused ion beam)fabricated micropillars. Here we utilize the model in Fig. 2.1(b) but with a $3^{\circ}$ taper, and observe its deformation field. Figure 4.17 shows the contour plot of equivalent plastic strain at the macroscopic compressive strain of 0.042 , in the metallic glass of aspect ratio 1.0 above the Si base. It can be seen that localized deformation occurs primarily in the upper region away from the interface. In addition to the interfacial constraint, the smaller cross section area near the top boundary results in stress concentration, which also contributes to the inhomogeneous distribution of plastic strain. Constrained deformation due to physical confinement is thus further enhanced by the geometrical effect.

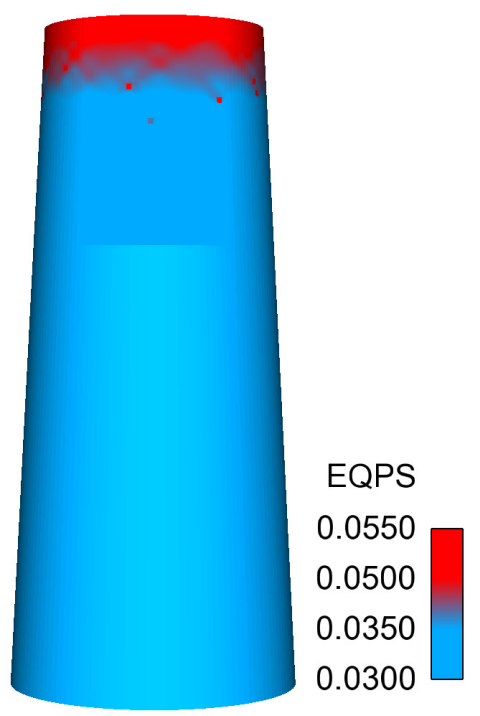

Figure 4.17: Contour plot of equivalent strain in the Si-base attached metallic glass model, with a $3^{\circ}$ taper, at the macroscopic compressive strain of 0.042 . 


\subsection{Four Point Bending}

A common experiment perfomed during testing of metallic glasses in both bulk and thin layer form is a simple four point bending test. Experimetal findings have shown that the formation of localized shear bands can lead to catastropic failure of the specimen under bending [32]. In order to further study the formation of shear bands in mealltic glass a four point bending simulation was run. The beam subjected to four point bending in Figure 4.18 is now considered. Unlike the micro pillars considered early the shear bands are not uniformly distriubuted and tend to form around the top and bottom faces of the beam. The plastic strains inside the shear bands are still much greater than the surrounding material.

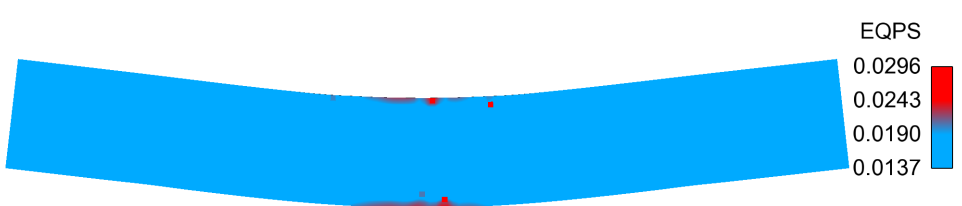

(a)

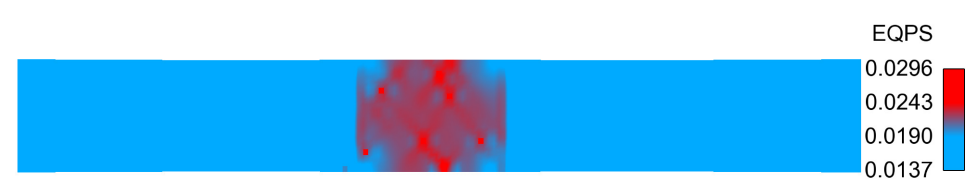

(b)

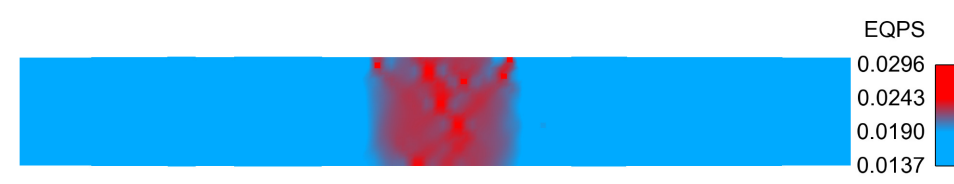

(c)

Figure 4.18: Contour plots of equivalent plastic strain in metallic glass due to bending deformtation: (a) Front view (b) Top view (c) Bottom view. 
Chapter 4. Results and Discussion

\subsubsection{Comparison with Experimentation}

Figure4.19 shows the results of an experimental bending test performed on a specimen consiting of a MG/Ti bilayered-coated with BMG. Figure4.19(a) shows that shear bands have formed on the tensile surface during deformation. The formation of shear bands in the FEA results are in qualitative agreement with the experimental results. Further extensive modeling is needed to better understand the enhancment of bending ductility of metallic glasses.
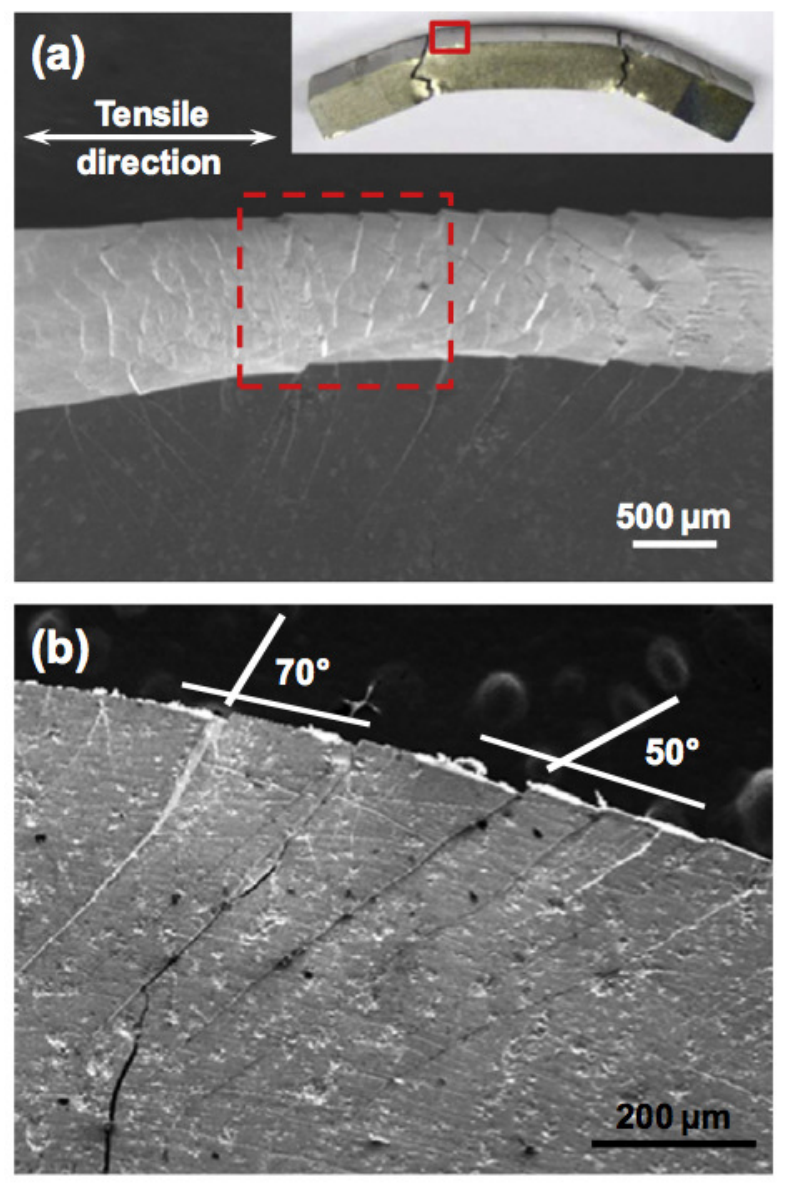

Figure 4.19: SEM micrographs of MG/Ti bilayer-coated BMG surface after bending to failure (a surface strain of 13.7\%): (a) 50 tilted and (b) side view. [32] 


\section{Chapter 5}

\section{Conclusions and Suggested Future Work}

In this research, systematic finite element analyses were conducted to study the evolution of shear bands in constrained metallic glass thin films. The models have the appearance of micropillars subject to compression, with or without a supporting base material, or with a multilayered configuration. Incorporation of randomized perturbation points in the model facilitated the localized plastic deformation. Salient findings from the modeling are summarized below.

- Without the influence of external constraint, the same stress-strain behavior and shear banding configuration can be obtained for stand-alone metallic glasses with various aspect ratios.

- When attached to a $\mathrm{Si}$ base (substrate), metallic glass cylinders with lower aspect ratios display higher plastic flow stresses. Shear bands are concentrated in the upper volume of the specimen, away from the interface with the base material. The effect is further enhanced if the cylinder shows a tapered geometry. 


\section{Chapter 5. Conclusions and Suggested Future Work}

- The effect of aspect ratio on the apparent yield stress for base-attached metallic glasses follows the same trend as reported in experiment. The yield stress stays nearly constant if the aspect ratio is greater than 0.5 .

- Substrate-induced constraint is found to influence overall plastic deformation behavior in fundamentally the same way, regardless of the localized or homogeneous nature of the deformation.

- Forming multilayers by bonding thin-film metallic-glass with crystalline interlayers (with traditional plastic behavior) can potentially alleviate the strongly heterogeneous deformation configuration.

The following items are suggested for future work:

- Adding contact defintions to the metallic glass silicon base interface could give more insight into the evolution of shear bands through the substrate.

- Further finite element analysis of metallic specimens coated with thin film metallic glasses in order to better understand the dramtic bending ductility enhancment.

- Alternative reference materials can be used in the multilayer case to further examine the potential to alleviate the strongly heterogeneous deformation configuration.

- Incorporating more complex physics such as element death into the models would give further insight on how shear band-induced failure developes in metallic glasses. 
Chapter 5. Conclusions and Suggested Future Work

\subsection{Implications}

The mechanical properties of metallic glasses have made them a promising material for a variety of engineering applications. Metallic glasses have great potential for use in structural and functional applications and are currently viewed as one of the most important metallic materials. Recent applications of MG's in mico- and nano- devices have helped spark intense research and may stimulate the development of new industries. This study continues to give support to previous experimental findings, which will allow future researchers to expand and innovate new experiments to explore the unquie charcteristics of metallic glasses. It is hoped that the results from this study will provide the material science community with further insight on the mechanical behavior of metallic glasses. 


\section{References}

[1] Y.-L. Shen. Constrained Deformation of Materials. Springer, 2010.

[2] J. C. Ye, J. P. Chu, Y. C. Chen, Q. Wang, and Y. Yang. Hardness, yield strength, and plastic flow in thin film metallic-glass. Journal of Applied Physics, 112:053516, 2012.

[3] J. P. Chu, J. C. Huang, J. S. C. Jang, Y. C. Wang, and P. K. Liaw. Thin film metallic glasses: preparations, properties, and applications. JOM, 62:19, 2010.

[4] A. Inoue. Stabilization of metallic supercooled liquid and bulk amorphous alloys. Acta Materialia, 48:279, 2000.

[5] J.F. Loffler. Bulk metallic glasses. Intermetallics, 11:529, 2003.

[6] W.H. Wang, C. Dong, and C.H. Shek. Bulk metallic glasses. Mater.Sci.Eng., $R, 44: 45,2004$.

[7] C.A. Schuh, T.C. Hufnagel, and U. Ramamurty. Mechanical behavior of amorphous alloys. Acta Materialia, 55:4067, 2007.

[8] M. Chen. Mechanical behavior of metallic glasses: Microscopic understanding of strength and ductility. Materials Research, 38:445, 2008.

[9] Y.Q. Cheng and E. Ma. Atomic-level structure and structureproperty relationship in metallic glasses. Progress in Materials Science, 56:379, 2011.

[10] A. Inoue and A. Takeuch. Recent development and application products of bulk glassy alloys. Acta Materialia, 59:2243, 2011.

[11] M. A. Meyers and K. K. Chawla. Mechanical Behavior of Materials. Cambridge University Press, second edition edition, 2009. 
References

[12] Ritupan Sarmah, G. Anathakrishna, B.A. Sun, and W.H. Wang. Hidden order in serrated flow of metallic glasses. Acta Materialia, 59:4482, 2011.

[13] W. Klement, R.H. Willens, and P.O.L. Duwez. Non-crystalline structure in gold-silicon alloys. Nature, 187:869, 1960.

[14] H.S. Chen and D. Turnbull. Formation, stability and structure of palladiumsilicon based alloy glasses. Acta Metallurgica, 17:1021, 1969.

[15] H.S. Chen. Thermodynamic considerations on the formation and stability of metallic glasses. Acta Metallurgica, 22:1505, 1974.

[16] A. Inoue, T. Zhang, and T. Masumoto. Zr-al-no amorphous alloys with high glass transition temperature and significant supercooled liquid region. Materials Transcations, 31:177, 1990.

[17] J. P. Chu, J. S. C. Jang, J. C. Huang, H. S. Chou, Y. Yang, J. C. Ye, Y. C. Wang, J. W. Lee, F. X. Liu, P. K. Liaw, Y. C. Chen, C. M. Lee, C. L. Li, and C. Rullyani. Thin film metallic glasses: Unique properties and potential applications. Thin Solid Films, 520:5097, 2012.

[18] B. E. Schuster, Q. Wei, M. H. Ervin, S. O. Hruszkewycz, M. K. Miller, T. C. Hufnagel, and K. T. Ramesh. Bulk and microscale compressive properties of a pd-based metallic glass. Scripta Materialia, 57:517, 2007.

[19] C. J. Lee, J. C. Huang, and T. G. Nieh. Sample size effect and microcompression of mg65cu25gd10 metallic glass. Applied Physics Letters, 91:161913, 2007.

[20] H. Guo, P. F. Yan, Y. B. Wang, J. Tan, Z. F. Zhang, M. L. Sui, and E. Ma. Tensile ductility and necking of metallic glass. Nature Materials, 6:735, 2007.

[21] Z. W. Shan, J. Li, Y. Q. Cheng, A. M. Minor, S. A. Syed Asif, O. L. Warren, and E. Ma. Plastic flow and failure resistance of metallic glass: Insight from in situ compression of nanopillars. Physical Review B, 77:155419, 2008.

[22] H. Lai, C.J. Lee, Y.T. Cheng, H.S. Chou, H.M. Chen, X.H. Du, C.I. Chang, J.C. Huang, S.R. Jian, J.S.C. Jang, and T.G. Nieh. Bulk and microscale compressive behavior of a zr-based metallic glass. Scripta Materialia, 58:890, 2008.

[23] C. A. Volkert, A. Donohue, and F. Spaepen. Effect of sample size on deformation in amorphous metals. Journal of Applied Physics, 103:083539, 2008.

[24] J. C. Ye, J. Lu, Y. Yang, and P. K. Liaw. Study of the intrinsic ductile to brittle transition mechanism of metallic glasses. Acta Materialia, 57:6037, 2009. 
References

[25] A. Bharathula, S.-W. Lee, W. J. Wright, and K. M. Flores. Compression testing of metallic glass at small length scales: Effects on deformation mode and stability. Acta Materialia, 58:5789, 2010.

[26] Y. Yang, J. C. Ye, J. Lu, P. K. Liaw, and C. T. Li. Characteristic length scales governing plasticity/brittleness of bulk metallic glasses at ambient temperature. Applied Physics Letters, 96:011905, 2010.

[27] C. E. Packard and C. A. Schuh. Initiation of a shear band near a stress concentration in metallic glass. Acta Materialia, 55:5348, 2007.

[28] Y.-L. Shen. Externally constrained plastic flow in miniaturized metallic structures: A continuum-based approach to thin films, lines, and joints. Progress in Materials Science, 53:838, 2008.

[29] A. Mendelson. Plasticity: Theory and Application. MacMillan, 1968.

[30] H. Kimura and T. Masumoto. Amorphous Metallic Alloys. Butterworth, 1983.

[31] H. A. Bruck, T. Christman, A. J. Rosakis, and W. L. Johnson. Quasi-static constitutive behavior of zr41.25ti13.75ni10cu12.5be22.5 bulk amorphous alloys. Scripta Metallurgica et Materialia, 30:429, 1994.

[32] J. P. Chu, J. E. Greene, J. S. C. Jang, J. C. Huang, Y.-L. Shen, P. K. Liaw, Y. Yokoyama, A. Inoue, and T. G. Nieh. Bendable bulk metallic glass: Effects of a thin, adhesive, strong, and ductile coating. Acta Materialia, 60:3326, 2012.

[33] J. Reddy. An Introduction to the Finite Element Method. McGraw-Hill Mechanical Engineering. McGraw-Hill Education, 2005.

[34] Vince Adams. A Designer's Guide to Simulation with Finite Element Analysis. NAFEMS, 2008.

[35] Y.-L. Shen, M. Finot, A. Needleman, and S. Suresh. Effective plastic response of two-phase composites. Acta Metallurgica et Materialia, 43:1701, 1995.

[36] Y.-L. Shen and Y. L. Guo. Indentation modeling of heterogeneous materials. Modelling and Simulation in Materials Science and Engineering, 9:391, 2001.

[37] M. C. Liu, C. J. Lee, Y. H. Lai, and J. C. Huang. Microscale deformation behavior of amorphous/nanocrystalline multilayered pillars. Thin Solid Films, 518:7295, 2010.

[38] H. S. Chou, X. H. Du, C. J. Lee, and J. C. Huang. Enhanced mechanical properties of multilayered micropillars of amorphous zrcuti and nanocrystalline ta layers. Intermetallics, 19:1047, 2011. 
References

[39] S. Y. Kuan, H. S. Chou, M. C. Liu, X. H. Du, and J. C. Huang. Micromechanical response for the amorphous/amorphous nanolaminates. Intermetallics, 18:2453, 2010 . 